\title{
Transcriptome Analysis of Pepper (Capsicum annuum) Revealed a Role of 24-Epibrassinolide in Response to Chilling
}

\begin{abstract}
Jie $\mathrm{Li}^{1+}$, Ping Yang ${ }^{2+}$, Jungen Kang ${ }^{3}$, Yantai Gan ${ }^{4,5}$, Jihua $\mathrm{Yu}^{1}$, Alejandro Calderón-Urrea ${ }^{6}$, Jian Lyu ${ }^{1}$, Guobin Zhang ${ }^{1}$, Zhi Feng ${ }^{1}$ and Jianming Xie ${ }^{1 *}$

${ }^{1}$ Department of Facility Horticulture Science, College of Horticulture, Gansu Agricultural University, Lanzhou, China, ${ }^{2}$ Department of Crop Cultivation and Farming System, College of Agronomy, Gansu Agricultural University, Lanzhou, China, ${ }^{3}$ Department of Vegetable Genetics and Breeding, Beijing Vegetable Research Center, Beijing Academy of Agriculture and Forestry Sciences, Beijing, China, ${ }^{4}$ Semiarid Prairie Agricultural Research Centre, Agriculture and Agri-Food Canada, Swift Current, SK, Canada, ${ }^{5}$ Gansu Provincial Key Lab of Aridland Crop Science, Gansu Agricultural University, Lanzhou, China, ${ }^{6}$ Department of Biology, California State University Fresno, Fresno, CA, USA
\end{abstract}

OPEN ACCESS

Edited by:

Claudio Bonghi, University of Padua, Italy

Reviewed by: Antonio Ferrante, University of Milan, Italy

Rui Fan,

Chinese Academy of Tropical Agricultural Science, China

*Correspondence: Jianming Xie gsau23@163.com

${ }^{\dagger}$ These authors have contributed equally to this work.

Specialty section:

This article was submitted to Crop Science and Horticulture, a section of the journal Frontiers in Plant Science

Received: 10 May 2016 Accepted: 11 August 2016

Published: 29 August 2016

Citation:

Li J, Yang P, Kang J, Gan Y, Yu J, Calderón-Urrea A, Lyu J, Zhang G,

Feng $Z$ and Xie $J$ (2016)

Transcriptome Analysis of Pepper (Capsicum annuum) Revealed a Role of 24-Epibrassinolide in Response to

Chilling. Front. Plant Sci. 7:1281.

doi: 10.3389/fpls.2016.01281
Brassinosteroids (BRs) have positive effects on many processes during plant growth, development, and various abiotic stress responses. However, little information is available regarding the global gene expression of BRs in response to chilling stress in pepper. In this study, we used RNA sequencing to determine the molecular roles of 24-epibrassinolide (EBR) during a chilling stress response. There were 39,829 transcripts, and, among them, 656 were differently-expressed genes (DEGs) following EBR treatment (Chill+EBR) compared with the control (Chill only), including 335 upregulated and 321 down-regulated DEGs. We selected 20 genes out of the 656 DEGs for RT-qPCR analysis to confirm the RNA-Seq. Based on GO enrich and KEGG pathway analysis, we found that photosynthesis was significantly up-enriched in biological processes, accompanied by significant increases in the net photosynthetic rate (Pn), $\mathrm{Fv} / \mathrm{Fm}$, and chlorophyll content. Furthermore, the results indicate that EBR enhanced endogenous levels of salicylic acid (SA) and jasmonic acid (JA) while suppressing the ethylene $(E T H)$ biosynthesis pathway, suggesting that BRs function via a synergistic cross-talk with SA, JA, and ETH signaling pathways in response to chilling stress. In addition, EBR induced cellulose synthase-like protein and UDP-glycosyltransferase, suggesting a contribution to the formation of cell wall and hormone metabolism. EBR also triggered the calcium signaling transduction in cytoplasm, and activated the expression of cellular redox homeostasis related genes, such as GSTX1, PER72, and CAT2. This work, therefor, identified the specific genes showed different expression patterns in EBR-treated pepper and associated with the processes of hormone metabolism, redox, signaling, transcription, and defense. Our study provides the first evidence of the potent roles of BRs, at the transcription level, to induce the tolerance to chilling stress in pepper as a function of the combination of the transcriptional activities, signaling transduction, and metabolic homeostasis.

Keywords: pepper, Brassinosteroid, chill-stress, transcriptome, RNA sequencing 


\section{INTRODUCTION}

Chilling stress affects the geographical distribution of many important vegetables such as pepper (Capsicum annuum L.; Sanghera et al., 2011). This abiotic stress adversely affects plant growth and development and the yield and quality of many crops (Janská et al., 2010; Ren et al., 2014). In Northern China, where greenhouse cultivation is used for vegetable production, it is very common for plants suffer a chilling injury during the fall-towinter transition period when a sudden temperature drops often leads to early frost damage. In vegetables, low temperatures lead to the arrest of growth or abortion of flower buds, resulting in significant yield and economic losses (Chinnusamy et al., 2007).

Plants have evolved pleiotropic and intricate regulatory functions to defend against environmental stresses (Xia et al., 2009). Under stress, plants produce a number of phytohormones, such as salicylic acid (SA), brassinosteroids (BRs), and abscisic acid (ABA), which play a critical role in the perception of external signals and the activation of defense mechanism within plants. Brassinosteroids, a group of naturally occurring plant steroids, have been shown to provide positive effects on the regulation of plant growth and a broad spectrum of physiological responses to abiotic stresses, such as high and low temperature stress (Mazorra et al., 2002; Bajguz, 2009), drought (Yuan et al., 2010), and salinity injury (Liu et al., 2014). For example, BRs increase thermotolerance of plants by inducing heat shock protein synthesis and gene expression for heat tolerance (Dhaubhadel et al., 2002; Dhaubhadel and Krishna, 2008). BRs induce cold-related gene expression in Brassica napus and Arabidopsis thaliana (Kagale et al., 2007). Ubiquitin-conjugating enzyme (UBC32) as a critical gene involved in BRI1 biosynthesis and ER-associated protein degradation $(E R A D)$ pathway positively regulates BR-induced salt tolerance (Cui et al., 2011). A synergistic interaction among $\mathrm{BR}$ signaling and the production of reactive oxygen species (ROS) induces the gene expression of respiratory burst oxidase homolog $(\mathrm{RBOH})$, whereas encoding NADPH oxidase and NADPH oxidase trigger apoplastic ROS accumulation, activating MAPKs to increase plant tolerance to stress (Hao et al., 2013).

RNA sequencing techniques have been used to investigate global expression profiles and reveal the signal transduction pathways involved in the resistance network under various stresses (Liu et al., 2015; Wang J. et al., 2015). The genome of pepper has been recently sequenced (Qin et al., 2014), which provides a valuable resource for molecular-based investigations for stress tolerance in plants. In previous studies, we found that exogenous BRs alleviated low temperature stress in pepper by enhancing antioxidant capacity and maintenance of photosystem II (Li et al., 2015a,b). However, it is unknown regarding the specific gene expression profile of BRs-induced chilling tolerance in pepper, and the genomic characteristics of the BRs-induced tolerance were undefined.

Here, we reveal the genes associated with chilling stress and the associated signaling pathways mediated by BRs using RNAseq analysis. The goals of the present study were to (i) provide insights into the pepper leaf transcriptome response to BRs under chilling stress; and (ii) uncover the genes and pathways that are associated with BRs-induced stress tolerance in pepper.

\section{MATERIALS AND METHODS}

\section{Plant Material and Stress Treatment}

Pepper seeds (cv. "Xiangyan NO.16") were germinated in the dark for $72 \mathrm{~h}$ at $28^{\circ} \mathrm{C}$ before being transplanted into plastic pots containing a mixture of vermiculite and peat $(1: 2, \mathrm{v}: \mathrm{v})$ for subsequent growth. The seedlings were grown in an intelligent greenhouse with $25 / 15^{\circ} \mathrm{C}$ (day/night), photon flux density of $350-400 \mu \mathrm{mol} \mathrm{m}^{-2} \mathrm{~s}^{-1}, 12-\mathrm{h}$ photoperiod, and relative humidity of $60-80 \%$.

We used six different concentrations $(0,10,1,0.1,0.01$, and $0.001 \mu \mathrm{M})$ to manipulate BRs levels in pepper plants as described by Li et al. (2015b). We used 0.1 $\mu \mathrm{M}$ EBR as an optimum EBR concentration based on the results of the prior experiment where various concentrations were studied and the optimum level was identified.

At the 6-7 leaf stage (50 days after planting), the seedlings were divided into the following two groups: one was sprayed with $0.1 \mu \mathrm{M}$ EBR solution, and the other was sprayed with the same volume of double distilled water. Twenty-four hours later, seedlings in both groups were transferred to a controlled growth chamber with temperature at $15 / 5^{\circ} \mathrm{C}$ (day/night), photon flux density at $100 \mu \mathrm{mol} \mathrm{m} \mathrm{m}^{-2} \mathrm{~s}^{-1}, 12$-h photoperiod, and relative humidity of $80 \%$. Each treatment was replicated three times at the growth chamber, and each replicate had 20 plants. At day 7 of chilling, we collected two biological replicates of each treatment for sequencing, and each biological replicate had three plants. All samples were collected at the same time, ground into powder in liquid nitrogen and stored at $-80^{\circ} \mathrm{C}$ for further use.

These samples were labeled Chill+EBR (EBR treatment under chilling stress) and Chill (control).

\section{RNA Extraction and RNA-seq}

Total RNA was extracted from the Chill and Chill+EBR samples (leaves) with Trizol reagent (Invitrogen, Carlsbad, CA, USA) using method described by $\mathrm{Hu}$ et al. (2012). The total RNA extraction was divided into two aliquots; one aliquote was used for RNA-sequencing, and the other was used for real-time PCR. The RNA-sequencing was performed using an Illumina HiSeq 4000 platform (Illumina, San Diego, CA, USA) at Novogene Bioinformatics Technology Co., China. Each sample generated more than 6 gigabyte of data. The clean reads were filtered from raw sequencing data and the low-quality reads containing unknown nucleotides or adaptor sequences were removed; this procedure was performed in accordance with the method of Chen et al. (2014). The filtered clean reads were aligned to C. annuum reference genome (http://peppersequence.genomics.cn/page/species/download.jsp).

\section{Differential Expression Analysis}

We performed differential expression analysis for both Chill and Chill+EBR treatments based on the DESeq R package, which allowed for statistical analysis using the negative binomial distribution model (Wang et al., 2010). To control the false 
discovery rate, we adjusted the resulting $p$-values according to Benjamini and Hochberg's approach (Benjamini and Hochberg, 1995), where an adjusted $p<0.05$ is accepted to represent differentially expressed genes (DEGs).

We performed gene ontology (GO) enrichment analysis of the DEGs according to the GOseq $\mathrm{R}$ package, and GO terms with $q<0.05$ were regarded as significantly enriched (Young et al., 2010). We carried out the statistical enrichment of the differential expression genes in Kyoto Encyclopedia of Genes and Genomes (KEGG) pathways using KOBAS software (Xie et al., 2011).

\section{Validation of DEGs by Real-Time Quantitative PCR}

Twenty transcript genes were selected for the qRT-PCR assay; the genes and gene-specific primers used are summarized in Table S1. Actin was used as an internal reference. qRT-PCR was performed using SYBR-Green (ABI-Invitrogen, California, USA) on an ABI 7900 Fast Real-Time PCR Detection System (Applied Biosystems, Carlsbad, USA). A real-time RT-PCR reaction (20 $\mu \mathrm{l}$ ) included $10 \mu \mathrm{l}$ of $2 \times$ SuperReal PreMix Plus, $2 \mu \mathrm{l}$ cDNA, $1 \mu \mathrm{l}$

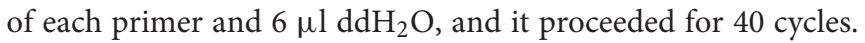
The relative expression levels of the selected 20 genes normalized to the expression level of actin (internal reference control) were calculated from cycle threshold values using the $2^{-\Delta \Delta \mathrm{Ct}}$ method (Livak and Schmittgen, 2001).

\section{Chlorophyll Content and Net Photosynthetic Rate}

The chlorophyll $\mathrm{a}$ and $\mathrm{b}$ concentrations were determined according to the method of Arnon (1949). Net photosynthetic rate $(\mathrm{Pn})$ in the fully expanded leaves of pepper plants was measured by Ciras-2 portable photosynthesis system (PP Systems, USA).

\section{Chlorophyll Fluorescence Determination}

The chlorophyll fluorescence parameters in pepper leaves were measured using a pulse-modulated fluorometer (FMS-2,
Hansatech, Norfolk, UK). Pepper seedlings were placed in the dark for $30 \mathrm{~min}$ and were prepared for the determination of the $\mathrm{Fv} / \mathrm{Fm}$.

\section{Analysis of Hormones and Hormone Metabolites}

Pepper leaf tissues treated with $\mathrm{DDH}_{2} \mathrm{O}$ or $0.1 \mu \mathrm{M}$ EBR under chilling stress were collected in liquid nitrogen and stored at $-80^{\circ} \mathrm{C}$. For analysis of IAA and ETH metabolites, SA and JA, leaf tissues were lyophilized using a freeze dryer; these procedures were in accordance with the methods of published research (Chiwocha et al., 2003; Peng and Zhou, 2009). Indole acetic acid oxidase (IAAO) activity was measured on acetone extract leaves by measuring residual IAA following incubation and agitation in the dark at $30^{\circ} \mathrm{C}$. One unit of IAAO activity was expressed as $1 \mathrm{mg}$ of IAA destroyed per milligram of protein per minute. ACC content and ACC synthase (ACS) activity was determined according to the method of Prasad and Cline (Prasad and Cline, 1987).

\section{RESULTS}

\section{Mapping and Quantitative Assessment of liiumina Sequence}

We constructed two libraries from Chill and Chill+EBR for RNA-Seq. A total of 32.49 million (Chill) and 39.86 million (Chill+EBR) reads were generated. After removing low-quality regions, adapters, and possible contamination, we obtained more than 3 giga base clean bases with a Q20 percentage over 92\%, Q30 percentage over 86\%, and a GC percentage between 42.9 and 43.7\% (Table 1).

Each library that produced the clean reads was aligned to the recently released C. annuum reference genome, release_2.0 (Qin et al., 2014). The proportion of clean reads in the two pepper transcriptome libraries that mapped to $C$. annuum reference genome ranged from 85.34 to $87.60 \%$ (Table 2). A total of 39,829 genes were confirmed from the mapped libraries, including the

\begin{tabular}{|c|c|c|c|c|c|c|c|}
\hline Sample name ${ }^{a}$ & Raw reads & Clean reads & Clean bases Gb & Error rate (\%) & $\mathrm{Q} 20^{\mathrm{b}}(\%)$ & Q30' $(\%)$ & GC content ${ }^{d}(\%)$ \\
\hline Chill1-1 & $33,040,705$ & $32,108,541$ & 4.01 & 0.04 & 94.63 & 89.54 & 42.91 \\
\hline Chill1-2 & $33,040,705$ & $32,108,541$ & 4.01 & 0.04 & 93.08 & 87.4 & 42.92 \\
\hline Chill2-1 & $31,938,987$ & $30,969,153$ & 3.87 & 0.04 & 94.44 & 89.22 & 43.38 \\
\hline Chill2-2 & $31,938,987$ & $30,969,153$ & 3.87 & 0.04 & 92.48 & 86.51 & 43.41 \\
\hline Chill+EBR1-1 & $41,024,790$ & $40,319,076$ & 5.04 & 0.04 & 94.22 & 88.8 & 43.63 \\
\hline Chill+EBR1-2 & $41,024,790$ & $40,319,076$ & 5.04 & 0.04 & 92.22 & 86.11 & 43.71 \\
\hline Chill+EBR2-1 & $38,701,387$ & $37,690,638$ & 4.71 & 0.04 & 95.02 & 90.2 & 42.95 \\
\hline Chill+EBR2-2 & $38,701,387$ & $37,690,638$ & 4.71 & 0.04 & 92.44 & 86.35 & 42.97 \\
\hline
\end{tabular}

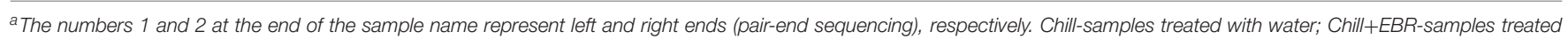
with $0.1 \mu \mathrm{M} E B R$.

${ }^{b}$ Percentage of bases with a Phred value of at least 20.

${ }^{c}$ Percentage of bases with a Phred value of at least 30.

${ }^{d}$ Proportion of guanidine and cytosine nucleotides among total nucleotides. 
TABLE 2 | Number of reads sequenced and mapped to the pepper genome.

\begin{tabular}{|c|c|c|c|c|}
\hline Sample name & Chill1 & Chill2 & Chill+EBR1 & Chill+EBR2 \\
\hline Total reads & $64,217,082$ & $61,938,306$ & $80,638,152$ & $75,381,276$ \\
\hline Total mapped & $56,251,137$ (87.6\%) & $53,309,676$ (86.07\%) & $68,819,267$ (85.34\%) & $65,985,060$ (87.54\%) \\
\hline Multiple mapped & 2,129,037 (3.32\%) & $2,064,848$ (3.33\%) & $2,861,761$ (3.55\%) & $28,11,891$ (3.73\%) \\
\hline Uniquely mapped & $54,122,100$ (84.28\%) & $51,244,828$ (82.74\%) & $65,957,506$ (81.79\%) & 63173169 (83.8\%) \\
\hline Read-1 & $27,305,549$ (42.52\%) & $25,918,892$ (41.85\%) & $33,351,826(41.36 \%)$ & $32,030,587$ (42.49\%) \\
\hline Read-2 & $26,816,551$ (41.76\%) & $25,325,936$ (40.89\%) & 32605680 (40.43\%) & $31,142,582$ (41.31\%) \\
\hline Reads map to "+" & $27,008,832$ (42.06\%) & $25,526,323$ (41.21\%) & $32,830,110(40.71 \%)$ & $31,512,085$ (41.8\%) \\
\hline Reads map to “-" & $27,113,268$ (42.22\%) & $25,718,505$ (41.52\%) & 33127396 (41.08\%) & $31,661,084$ (42\%) \\
\hline Non-splice reads & $36,188,485$ (56.35\%) & 33853641 (54.66\%) & $43,682,428(54.17 \%)$ & $41,602,031$ (55.19\%) \\
\hline Splice reads & $17,933,615$ (27.93\%) & 17,391,187 (28.08\%) & $22,275,078$ (27.62\%) & $21,571,138$ (28.62\%) \\
\hline
\end{tabular}

\begin{tabular}{|c|c|c|}
\hline RPKM $^{\mathbf{a}}$ interval & Chill & Chill+EBR \\
\hline $0 \sim 1$ & $17,437(43.78 \%)^{b}$ & 17,267 (43.35\%) \\
\hline $1 \sim 3$ & 3859 (9.69\%) & 3925 (9.85\%) \\
\hline $3 \sim 15$ & 8283 (20.80\%) & 8415 (21.13\%) \\
\hline $15 \sim 60$ & 6932 (17.41\%) & 6954 (17.46\%) \\
\hline$>60$ & 3319 (8.33\%) & 3269 (8.21\%) \\
\hline
\end{tabular}

${ }^{a}$ Reads per kilo bases per million reads.

${ }^{b}$ Ratios of gene number to total gene number are presented in parentheses.

locations of exons and introns (Table S2). All of the RNAsequence data in this article have been deposited in the NCBISRA database and are accessible in SRX1959970.

\section{Transcriptome Profiles of the Leaves from the Two Groups of Pepper Seedlings}

The 39,829 genes from the mapped libraries were normalized (Table S3) using the reads per kilo bases per million reads (RPKMs) method (Mortazavi et al., 2008). With RPKMs in 0 to 1 , the genes were regarded as having a low expression level; genes with RPKMs of 3-15 were regarded as having a medium expression level; and genes with RPKM beyond 60 were regarded as having a very high expression level (Table 3).

\section{Differentially Expressed Genes in the Pepper Leaves}

We identified 656 DEGs between Chill and Chill+EBR treatments (Table S4; Figure 1A). We used hierarchical clustering of all the DEGs to observe the gene expression patterns, and it was evaluated by $\log _{10}$ RPKMs for the two groups (Figure 1B). Compared to Chill treatment, genes in Chill+EBR treatment contained 335 up-regulated genes and 321 down-regulated genes. These results suggest that EBR had a markedly effect on the transcription of a subset of genes response for chilling stress.

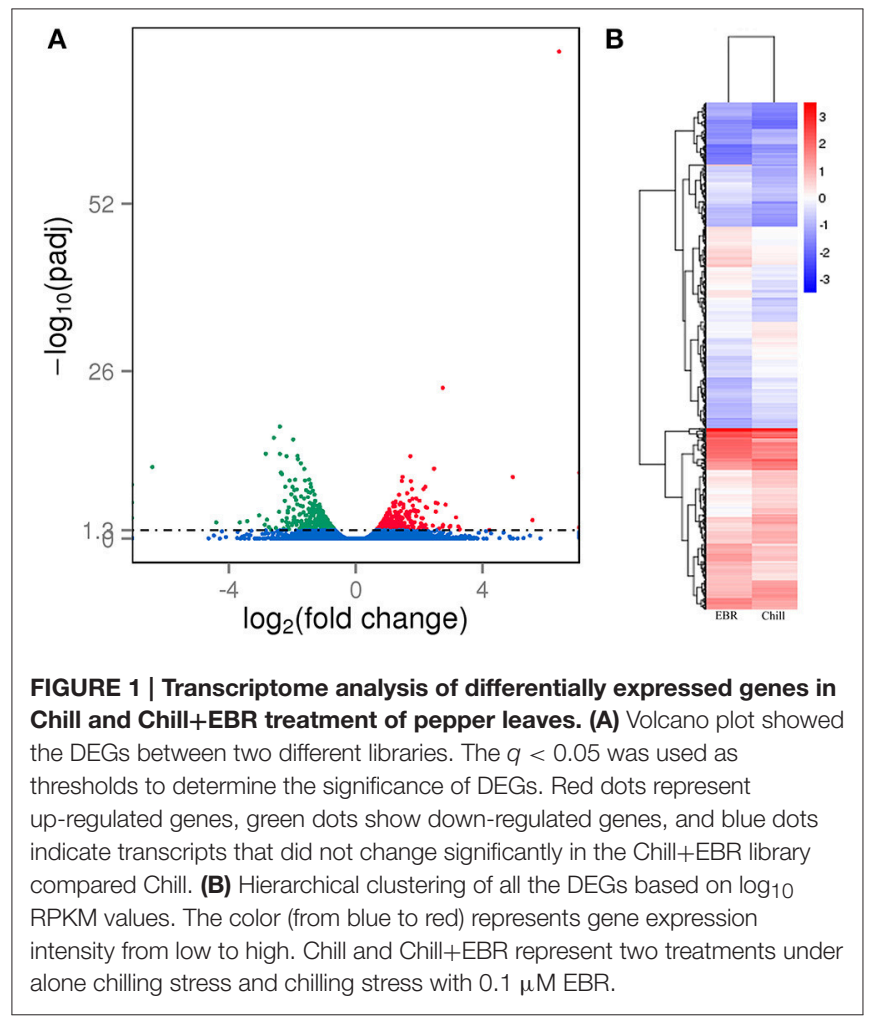

\section{Real-Time qPCR Analysis}

To validate the DEG data from RNA-sequencing, we randomly selected 20 DEG for qRT-PCR assay in EBR-mediated chilling stress. The qRT-PCR results showed a strong positive correlation with the RNA-seq $\left(R^{2}=0.947\right)$, indicating that the RNA-seq data were validated (Figure 2).

We evaluated the gene functions of DEGs using GO enrichment analysis, and it revealed the biological process, molecular function and cellular component categories for the 656 DEGs of the two groups tested (Table S5). A total of 335 up-regulated DEGs were enriched significantly in 10 functional terms. Among these, six terms were significantly enriched in cellular component, there was one term significantly enriched in 


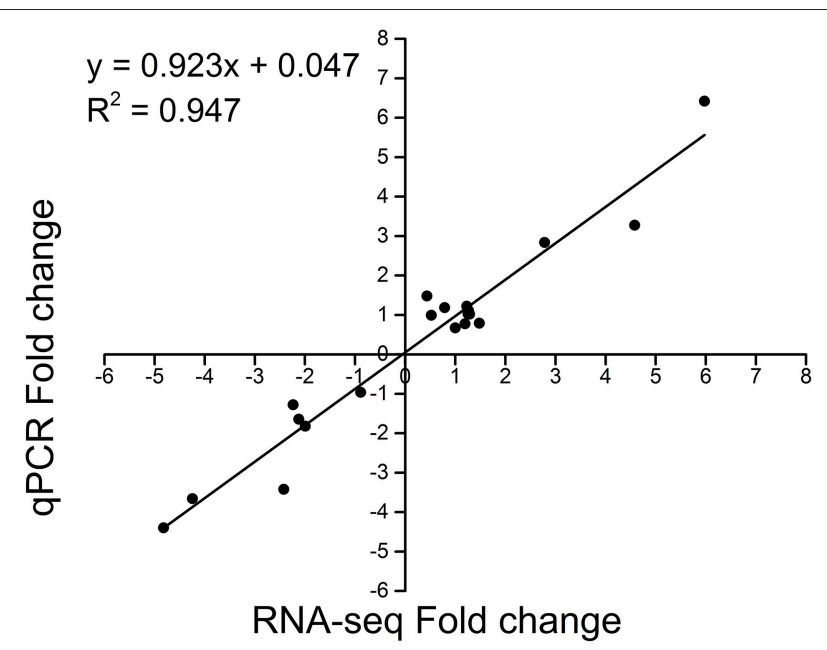

FIGURE 2 | Correlation of RNA-seq (y axis) and qRT-PCR data (x axis), and the assay is carried out for 20 randomly selected DEGs. GO and KEGG enrichment analyses.

molecular function, and two terms were significantly enriched in biological process. Within in the cellular component domain, the terms that were significantly enriched Photosystem I reaction center (GO: 0009538; 8 genes), photosystem (GO: 0009521; 15 genes), photosynthetic membrane (GO: 0034357; 15 genes), thylakoid (GO: 0009579;15 genes), thylakoid part (GO: 0044436; 15 genes), and photosystem I (GO: 0009522; 8 genes). Within the molecular function domain, the term that was significantly enriched intransferring hexosyl groups (GO: 0016758) with 16 genes. Within the biological process domain, the terms that were significantly enriched included Photosynthesis (GO: 0015979) and DNA-dependent transcription (GO: 0006352) with 19 and 9 genes, respectively. For down-regulated DEGs, three terms were markedly enriched in molecular function, and 41 terms were markedly enriched in biological process (Figure 3).

Among DGEs of the two groups tested the following 9 pathways, with a KEGG pathway annotation, were affected: carbon fixation in photosynthetic organisms, photosynthesis, photosynthesis-antenna proteins, carbon metabolism, pentose phosphate pathway, glyoxylate and dicarboxylate metabolism, metabolic pathways, zeatin biosynthesis, glycine, serine, and threonine metabolism pathway $(q<0.05$; Table S6; Figure 4).

\section{Effect of EBR on Chlorophyll Content and Net Photosynthetic Rate}

The exposure to chilling stress treatment influenced the morphological traits in pepper seedlings, and EBR application significantly alleviated the inhibited growth (Figure 5A). The net photosynthetic rate $(\mathrm{Pn})$ was increased significantly 7 days after EBR-treatment compared to without EBR; this was accompanied by a remarkable increase in chlorophyll content (Figures 5D,E). EBR (Chill+EBR) attenuated the inhibitory effect of chilling on photosynthesis. Low temperature treatments resulted in the reduction of $\mathrm{Fv} / \mathrm{Fm}$ in pepper seedlings, while the application of EBR significantly increased Fv/Fm (Figure 5C).

\section{Transcriptome Profiles of Photosynthesis-Related Genes}

The genes involved in the photosynthesis in the EBR-treated chilling responses were all up-regulated, and among them 8 genes encoded photosystem I reaction center subunit. It is known that the chlorophyll a/b-binding protein is an important component of light-harvesting complex II (Jansson, 1999, 1994). We found that EBR induced the expression of 10 genes encoding chlorophyll a/b-binding protein. ATP-dependent zinc metalloproteases, known to take part in chloroplast protein modification and the metabolism of extracellular matrix, were also up-regulated by EBR. Additionally, the genes involved in ATP synthase chain, oxygen-evolving enhancer protein, $\mathrm{ABC}$ transporter I family member, thylakoid lumenal protein, and PsbP domain-containing protein were all up-regulated, suggesting that BRs might play a significant and positive role in the photosynthesis processes under chilling stress (Table 4).

\section{Effect of EBR on the Endogenous Levels of Other Hormones and Their Metabolites}

To determine whether BR influenced the endogenous levels of other hormones, we assessed the concentrations of IAA, $\mathrm{ABA}, \mathrm{JA}$, and SA and ETH metabolites in the leaves of pepper seedlings grown in the absence or presence of $0.1 \mu \mathrm{M}$ EBR under chilling stress. The concentrations of ABA, SA and JA were significantly increased by $37.5,189.1$, and $132.3 \%$, respectively, in the EBR-treated seedlings compared to the untreated seedlings (Figure 6A). There was an increase in the level of IAA, but a significant decrease in the activity of acetic acid oxidase (IAAO) in the EBR-treated tissue (Figure 6B). There was a significant increase in the activity of ACC synthase (ACS) in the EBR-treated seedlings compared to chilling stress without EBR (Figure 6B).

\section{Transcriptome Profiles of Hormone Metabolism and Signaling-Related Genes}

EBR treatment under chilling stress induced changes in the expression of some genes involved in auxin signaling pathways, including auxin-induced protein, auxin-responsive protein, and auxin-binding protein (Table 5). Genes involved in cytokinin dehydrogenase, which plays a significant role in maintaining the well-organized cytokinin functions (Werner et al., 2003), were down-regulated by EBR in pepper. Protein phosphatase type 2C24 (P2C24) was identified as the second component of the ABA signaling pathway, which was also down-regulated by EBR. JA biosynthesis gene, linoleate 13S-lipoxygenase 2-1, and SA-related isochorismate synthase gene were all up-regulated, while four gibberellin-responsive genes were down-regulated. EBR treatment under chilling stress down-regulated all of the genes in the ETH signaling pathways. These genes included the ETH biosynthesis gene ACC synthase (ACS1), the induction of the ETH receptor (ETR), 8 ETH responsive transcription factors and one ETH insensitive 3-like 1 protein.

Late embryogenesis abundant protein D-29, which is involved in the tolerance to water stress resulting from desiccation or chilling injury was down-regulated by EBR. The genes involved in 
A

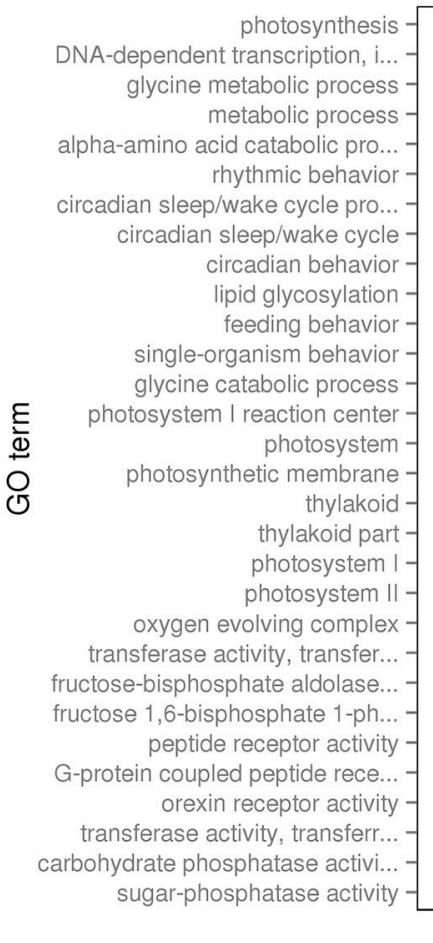

B

regulation of transcription, D... regulation of RNA metabolic pr.. regulation of RNA biosynthetic... regulation of cellular macromo.. regulation of biosynthetic pro... regulation of macromolecule bi.. regulation of cellular biosynt.. regulation of nucleobase-conta. regulation of nitrogen compoun.. regulation of gene expression regulation of macromolecule me... regulation of primary metaboli... regulation of cellular metabol... E nucleobase-containing compound.. () transcription, DNA-dependent Ð $\quad$ RNA biosynthetic process

regulation of cellular process aromatic compound biosynthetic.. organic cyclic compound biosyn... cellular nitrogen compound bio.. regulation of metabolic proces... regulation of biological proce... biological regulation macromolecule biosynthetic pro... biosynthetic process cellular macromolecule biosynt... nucleic acid binding transcrip... sequence-specific DNA binding ... sequence-specific DNA binding

The Most Enriched GO Terms

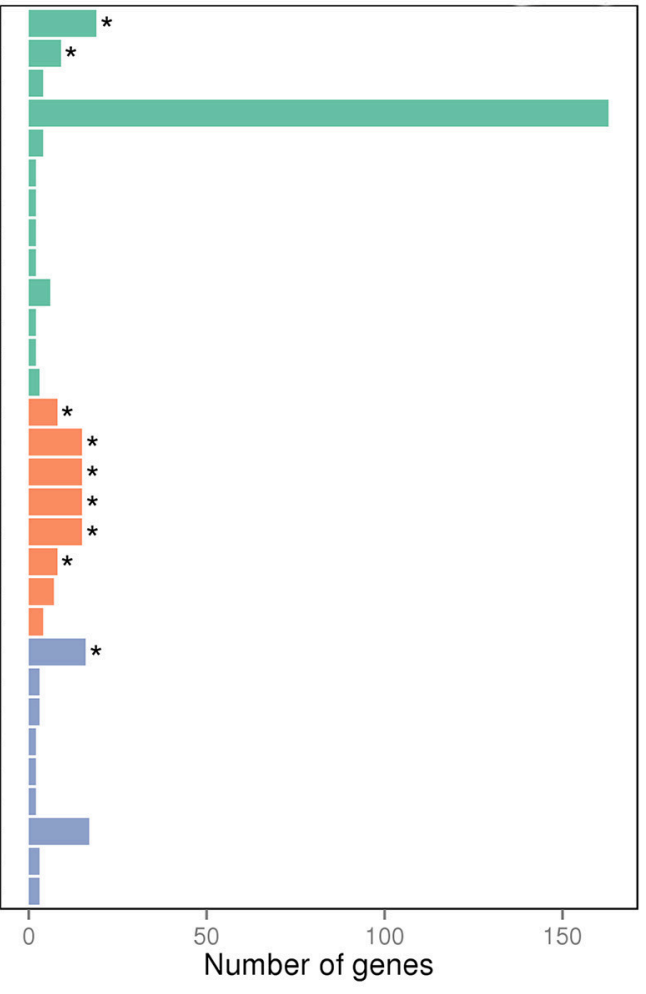

The Most Enriched GO Terms

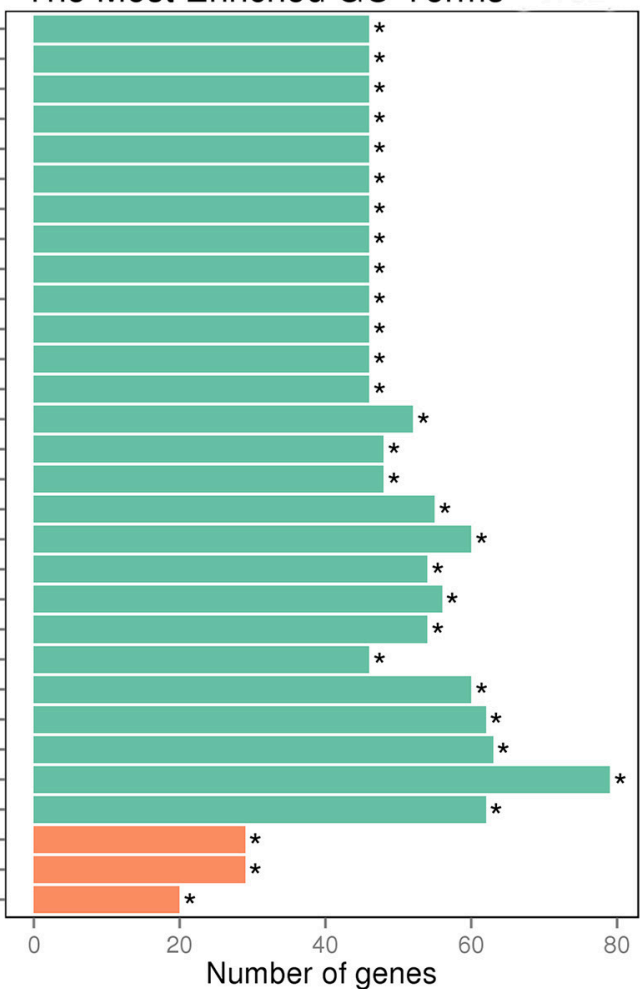

type

biological_process

cellular_component

molecular_function

FIGURE 3 | GO enrichment analysis of DEGs between Chill and Chill+EBR treatment. (A) up-regulation; (B) down-regulation. The 30 most enriched GO terms are shown. Asterisks indicate significantly enriched GO terms $(q<0.05)$. 


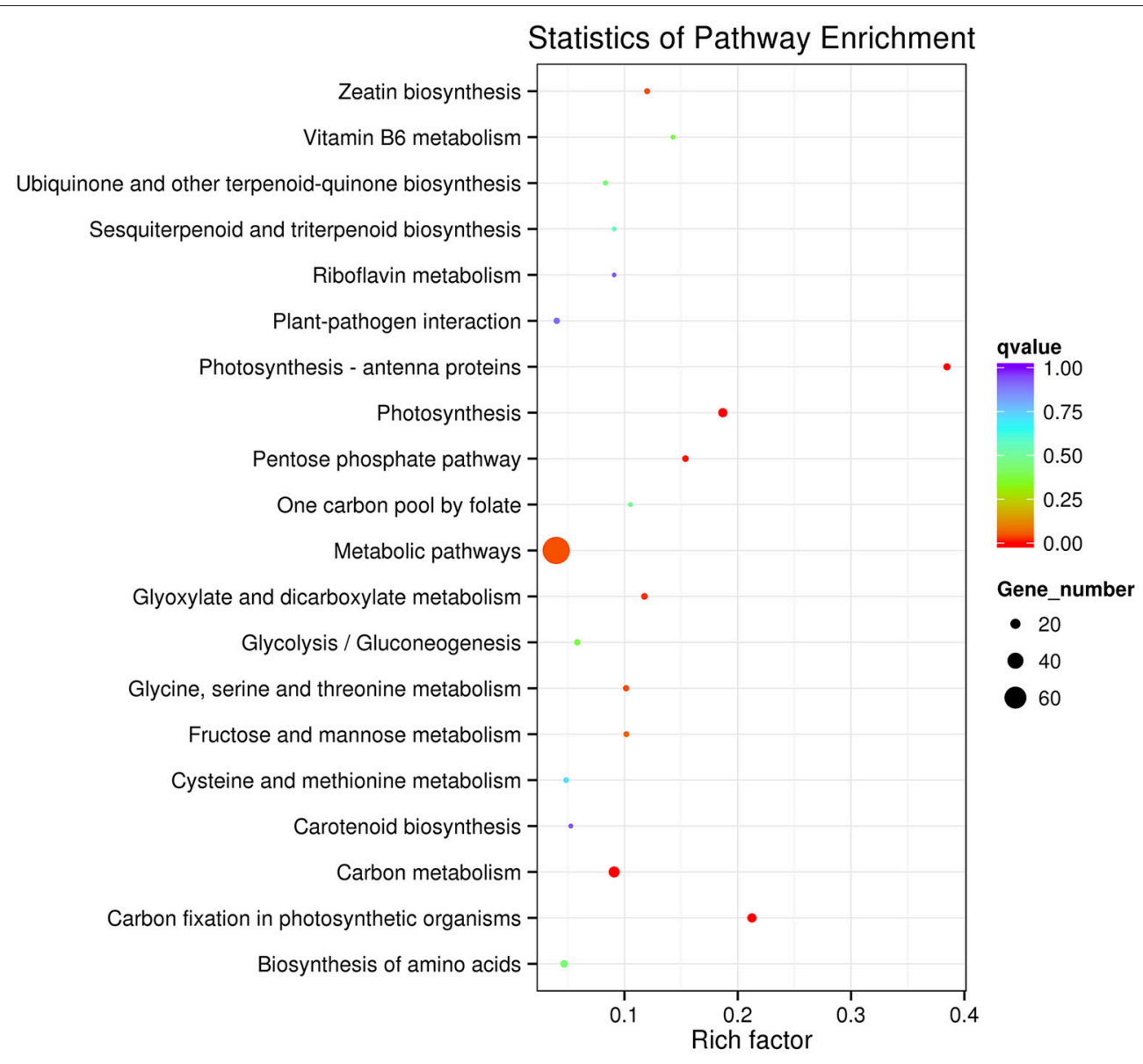

FIGURE 4 | KEGG pathway enrichment analysis of DEGs between Chill and Chill+EBR treatment. The left Y-axis shows the KEGG pathway. The X-axis shows the Rich factor. A high $q$-value is represented by blue and a low $q$-value is represented by red $(q<0.05)$.

proline responses including proline dehydrogenase and prolinerich receptor-like protein kinase PERK1 (PERK1) were downregulated, while PERK10 was up-regulated. $R B O H C$ which is associated with ROS production in plants, was down-regulated by EBR. There are three genes related to cysteine-rich receptor-like protein kinase, among them two genes were down-regulated, and the other gene was up-regulated. The RING-H2 finger protein ATL72 was up-regulated, while the RING-H2 finger protein ATL78 and RING-H2 finger protein 2 were down-regulated. The genes may be involved in the early steps of the plant defense signaling pathway. Dehydration-responsive elementbinding protein (DREB2C) and protein dehydration-induced 19 homolog (3DI19-3) which mediate cold-inducible transcription, were down-regulated by BRs (Table 5).

\section{Transcriptome Profiles of Calcium Signaling and Redox-Related Genes}

We found that EBR treatment under chilling stress up-regulated calcium-dependent protein kinase (CAS, Capana00g001365), while CBL-interacting serine/threonine-protein kinase, calcium uniporter protein, probable calcium-binding protein CML15, calcium-binding protein CML38, and calmodulin-related protein were down-regulated (Table 6).

All of the genes involved in redox homeostasis were up-regulated by EBR (Table 6). GSTX1, PER72, and CAT2 inactivate endogenous epoxides and hydroperoxides, and are associated with secondary metabolites during oxidative stress. CDSP32, which encodes thioredoxin as a physiological electron donor to the BAS1 peroxiredoxin, participated in the defense against lipid peroxidation in photosynthetic membranes (Broin and Rey, 2003). FAO4A was involved in the omega-oxidation pathway of lipid degradation. At1g06690 and $\mathrm{NAD}(\mathrm{P}) \mathrm{H}$ quinone oxidoreductase played a positive role in the antioxidant defense by generating vitamin $\mathrm{E}$ and ubiquinone.

\section{Transcriptome Profiles of Transcription Factors and Post Transcription}

We analyzed the function of transcription factors (TFs) in pepper based on their annotations in NCBI database. The majority of WRKY family TFs were down-regulated, such as WRKY11 [a negative regulator of resistance to Pst (Journot-Catalino et al., 

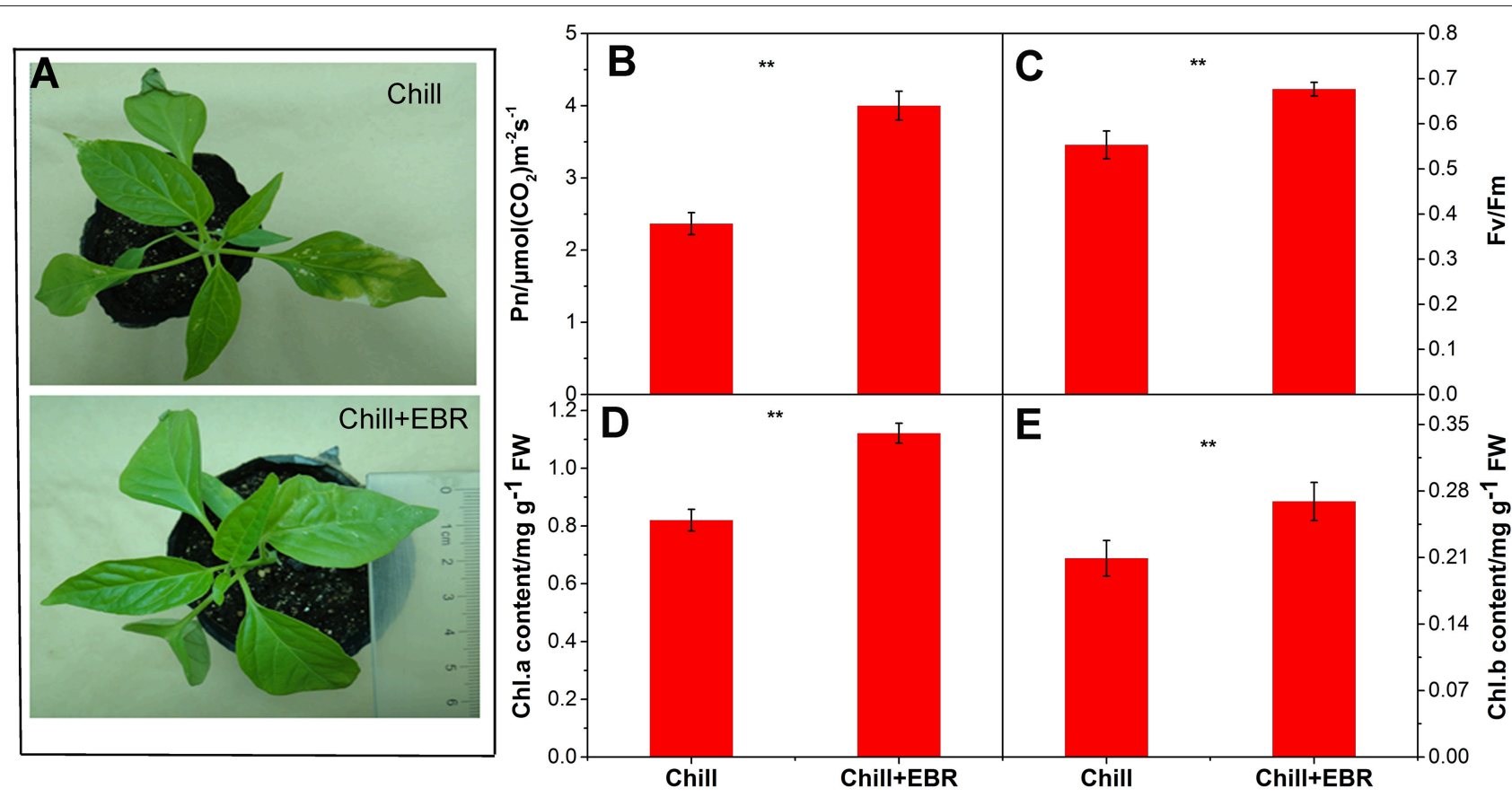

FIGURE 5 | Phenotypic changes (A), net photosynthetic rate (Pn) (B), Fv/Fm (C), chlorophyll a and chlorophyll b (D,E) in chill-stressed pepper seedlings with or without exogenous application of EBR. Asterisks above the histograms indicated significant differences between Chill and Chill + EBT by Student's $t$-test $\left({ }^{\star \star} P<0.01 ;{ }^{\star} P<0.05\right)$.

2006)], WRKY41, WRKY6, WRKY40 [a transcriptional repressor in plant cells response to abscisic acid and abiotic stress (Chen et al., 2010)], WRKY33, WRKY28, and WRKY20. Only WRKY51 (Capana12g001826), which positively mediated the signaling transduction of SA and JA (Gao et al., 2011), was up-regulated by EBR under chilling stress. The MYB family, which is involved in a diversity of gene regulation (Jackson et al., 1991), and NAC family members (NAC100, NAC002, and NAC072) were all downregulated by EBR under chilling stress. bHLH family members were up-regulated except $b H L H 130$. bHLH transcription factors positively regulated ABA-responsive kinase substrate (AKS) and facilitated stomatal opening by triggering the phosphorylation of AKS family transcription factors (Takahashi et al., 2013). Heatstress transcription factor A-2/B-1, the key regulator alleviating oxidative damage caused by heat stress negatively regulated by cold stress in Arabidopsis (Zhang et al., 2009), was downregulated by EBR in pepper. COL2, a circadian clock that controls many rhythmic processes (Kim et al., 2013), was up-regulated. $M G P$, which regulated tissue boundaries and asymmetric cell division and controlling SHORT-ROOT activity in a transcriptional and protein interaction network (Welch et al., 2007), was down-regulated by EBR. Additionally, ATHB7 mediates a drought response via transcriptional regulation in an ABA-dependent manner. ATHB-52 and ATHB-21, which responded to auxin, $\mathrm{ETH}$, and $\mathrm{ABA}$ or water deficits (Henriksson et al., 2005), were down-regulated by EBR. TCP19, involved in the orchestrated regulation of ICS1 expression (Wang X. et al., 2015), was down-regulated by EBR. GATA22, which participates in the GA-mediated signaling pathway (Richter et al., 2010) and chlorophyll biosynthetic process (Hudson et al., 2011), was up-regulated by EBR in pepper under chilling stress (Table 7).

EBR treatment induced CSLE6 and CSLH1 up-regulation, but down-regulated CSLG1, and it is known that they encode various non-cellulosic $\beta$-linked polysaccharides synthesis enzymes involved in the backbone of the cell wall (Doblin et al., 2009). Four genes encoding glycosyltransferase, which catalyzed the receptor substrates of cytokinin, auxin and ABA, were up-regulated. Similarly, three genes encoding UDP-glucose flavonoid 3-O-glucosyltransferase, GT6, GT7, and GT3, were upregulated by EBR (Table 7), and it is known that they are also involved in the detoxification of xenobiotics.

\section{DISCUSSION}

BRs, a group of naturally occurring plant steroids, are involved in many important cellular and physiological processes of plants in response to environmental stresses (Xia et al., 2009). Additionally, BRs mediate the response and signal transduction pathways of multiple hormones such as salicylic acid (SA), ethylene (ETH), jasmonic acid (JA), or abscisic acid (ABA) to abiotic stresses (U. K. Divi et al., 2010). In a previous study, we demonstrated that EBR treatment increased the basal chill tolerance of pepper via physiological and biochemical methods (Li et al., 2015a). To identify BR-mediated changes in gene expression, in the present study, we revealed the potent role of BRs in the response to chilling stress at the transcriptome level, and the findings provide the groundwork for elucidating the 
TABLE 4 | Photosynthesis related genes expression in pepper leaves as influenced by chilling alone or in combination EBR treatment.

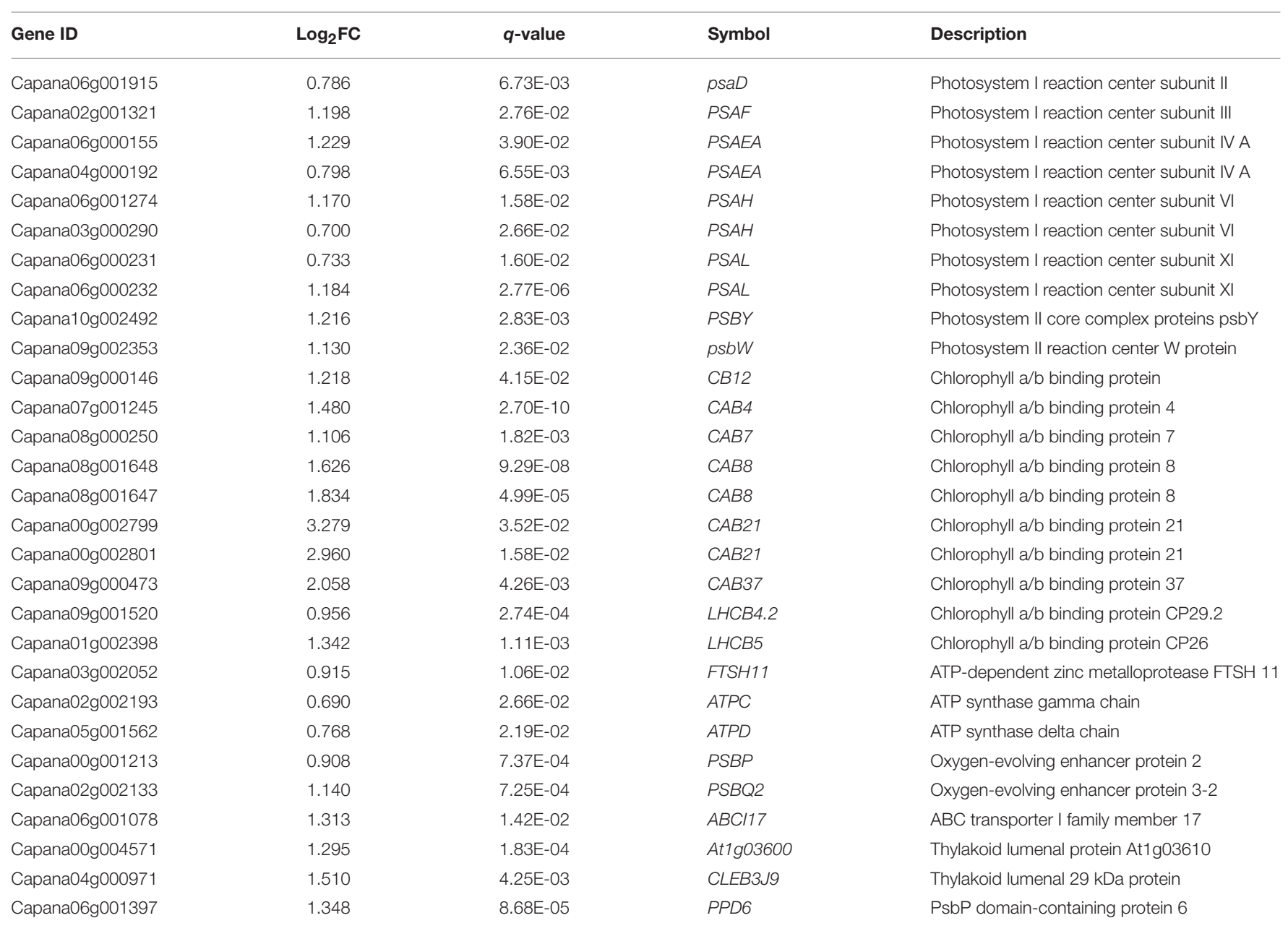

Shown are the q-value $(<0.05)$ for genes expression (comparison between EBR+Chill and Chill).

mechanism of BRs-induced chilling tolerance in pepper plants. We identified 656 DEGs, including 335 up-regulated and 321 down-regulated genes in the EBR-treated tissues compared to the chilling stress without EBR.

Photosynthesis is among the primary processes in plants that are often affected by chilling stress (Allen and Ort, 2001). In the present study, we used GO enrichment analysis and found that the 29 genes involved in photosynthesis were up-regulated by EBR under chilling stress conditions. Indeed, EBR-treated seedlings under chilling stress significantly enhanced net photosynthetic rate (Pn) and chlorophyll content when compared to controls (Figure 5). This suggests that BRs induction of chlorophyll and the net photosynthetic rate is a major factor contributing to the distinct photosynthetic characteristics in the BRs transcriptome. We identified several up-regulated genes associated with chloroplast organization (Capana02g002385) and the photosynthetic apparatus including the chloroplast and thylakoid luminal. In addition, we found that BRs also significantly increased Fv/Fm under chilling stress. These results suggest that EBR treatment contributes to the pepper leaves increased ability to absorb and transfer light energy in chilling conditions. Indeed, the 5 up-regulated genes were associated with redox regulation of photosystem II (Capana06g001397), transport activity (Capana06g001078, Capana03g002052), and photosystem II reaction center (Capana09g002353, Capana10g002492). KEGG pathway analysis also showed that the photosynthesis (ko00195) and photosynthesis-antenna proteins (ko00196) were significantly enriched up-regulated pathway terms (Figure 4).

As small molecules, plant hormones mediate many cellular processes in plants, including plant morphogenesis, and responses to changing environmental conditions (Kang et al., 2005; Pantin et al., 2013; Colebrook et al., 2014). Signal transduction components perceive plant hormone signals and transmit to the nuclear to induce gene expression synergistically with other signals to induce gene expression synergistically with other signals to influence response to environmental stress via a series of physiological processes ( $\mathrm{Lu}$ et al., 2014). In our study, some unigenes were markedly enriched in the hormone biosynthesis and signaling components processes. 


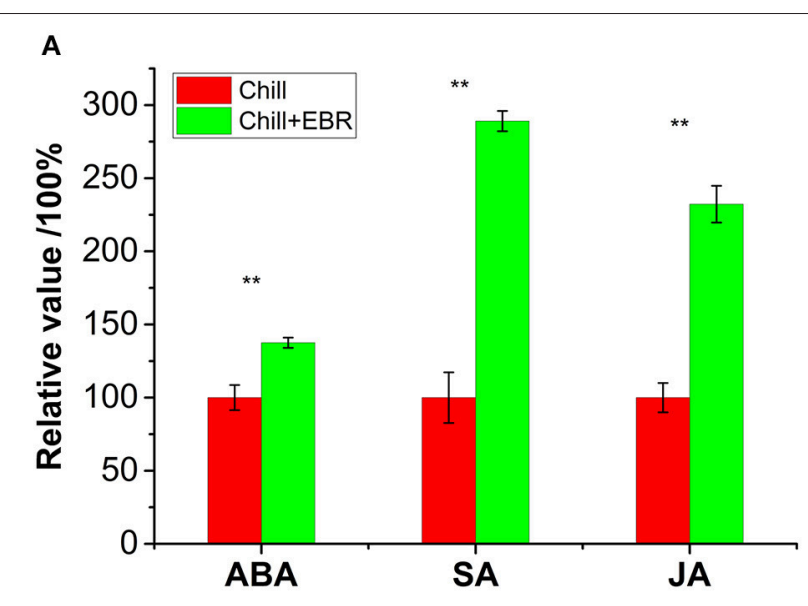

B

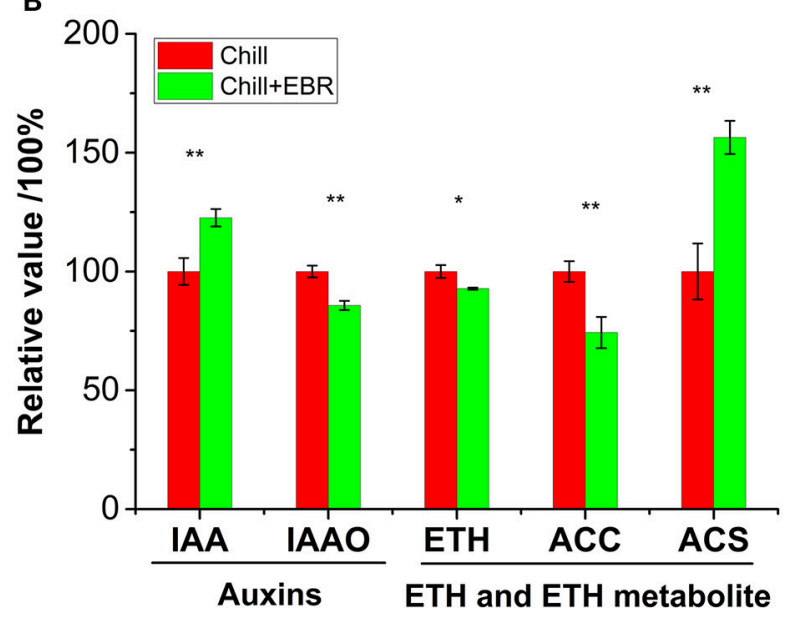

FIGURE 6 | Endogenous hormone profiles of pepper seedlings grown in the only chill stress (Chill) or chill stress with $0.1 \mu \mathrm{M}$ EBR

(Chill+EBR). (A) Contents of ABA, SA and JA in leaves of pepper seedlings. (B) Contents and metabolites of IAA and ETH in leaves of pepper seedlings. Error bars represent the standard error (SE) of the mean for three replicates. Asterisks above the histograms indicated significant differences between Chill and Chill + EBT by Student's $t$-test $\left({ }^{\star \star} P<0.01 ;{ }^{*} P<0.05\right)$.

These hormones including auxin, JA, ETH, ABA, and SA, and their signaling components were involved in BR-induced plant defense. Our results showed that the auxin, JA, and SA pathways appeared to act synergistically with BRs in mediating the response to chilling stress (Table 5). It is known that BRs can induce the SA-perceptive pathway or ABA-dependent pathways for resistance to heat and salt stress in A. thaliana or Chlorella vulgaris (Bajguz, 2009; Divi et al., 2010).

We demonstrated the effects of BRs on chilling tolerance via the transcriptome profiles of hormone metabolism and signalingrelated components in pepper. Accordingly, the transcription involved in hormone signaling components may function as important mediators of BR-induced resistance to chilling stress. We found that four DEGs were associated with auxin biosynthesis and signaling pathways, and the BRs mediated the down-regulation of auxin-induced protein $\mathrm{B} 6$ and IAA17. It is known that auxin-induced protein B6 induced the auxinactivated signaling pathway, whereas IAA17 functioned as a repressor of early auxin response genes (Liscum and Reed, 2002). Furthermore, we found that EBR-treated seedlings had significantly enhanced IAA content, but decreased the activity of acetic acid oxidase (IAAO). It was speculated that BRs reduced sensitivity to IAA by down-regulating genes encoding IAA conjugates. We found the down-regulation of genes involved in cytokinin; EBR treatment did not affect the endogenous levels of cytokinins (data not shown), but it induced cytokinin signaling in pepper. We speculated that BRs have a negative feedback loop to ensure the proper regulation of cytokinin functions, supported by previous observations (Werner et al., 2003). In addition, we found that ABA levels were increased by $37.5 \%$ in EBR-treated seedlings (Figure 6). Intriguingly, the gene $P 2 C 24$, encoding the second component of the ABA signaling pathway, was down regulated. Indeed, Yu et al. (2011) demonstrated some $\mathrm{ABA}$ response genes as the direct targets of BES1 in ChIP-chip analysis. Additionally, BRs can inhibit ABA effects. For example, BRs acted as an opposing factor, providing inhibitory effects of $\mathrm{ABA}$ on seed germination (Divi and Krishna, 2010). The molecular mechanism is that BRs inhibit BRASSINOSTEROID INSENSITIVE2 (BIN2), thus, repressing the BIN2-ABI5 (ABSCISIC ACID INSENSITIVE5) cascade and antagonizing ABA inhibitory effect on germination ( $\mathrm{Hu}$ and $\mathrm{Yu}$, 2014).

We found that JA and SA levels were significantly higher in the EBR-treated seedlings (Figure 6). Likewise, the JA biosynthesis gene, linoleate $13 \mathrm{~S}$-lipoxygenase 2 -1, and SA-related gene, isochorismate synthase (ICS), were up-regulated. These results suggest that the possible cross-talk of BRs with JA and SA signaling pathways plays a positive role of mediating chilling stress responses in pepper. In the present study, we found that EBR treatment decreased ETH levels, reduced the content of ACC which is an ETH synthetic substance, and increased ACS activity. We also identified genes encoding ETH signaling components (ACS1) and encoding ETH-insensitiive 3like protein and ethylene-responsive transcription factors $(E R F)$, which were down-regulated by EBR. This finding indicates that BRs and ETH have an antagonistic relationship under chilling stress and that ETH contributes to the activation of the BR signaling pathway and increased chill tolerance.

As secondary signaling molecules, ROS and calcium are crucial for plant defense against abiotic stresses. Here, we found that EBR up-regulated genes associated with cellular redox homeostasis, including glutathione S-transferase (GSTX1), peroxidase, catalase isozyme, and ferredoxin related genes (Table 6). It was reported that $\mathrm{RBOHs}$ are associated with ROS production in plants (Marino et al., 2012). We found that BRs triggered $R B O H C$ down-regulation in pepper, which suggested that BRs reduced apoplastic ROS accumulation generated by NADPH oxidase so that the plants increased stress tolerance. Calcium is a key element in many cellular processes in plants, and calcium signaling is crucial for plant defense against abiotic stresses (Yuan et al., 2007; Boudsocq and Sheen, 2013). In our study, EBR up-regulated CAS, which modulated cytoplasmic $\mathrm{Ca}^{2+}$ concentrations for the 
TABLE 5 | Hormone metabolism and signaling-related gene expression in pepper leaves as influenced by chilling alone or in combination EBR treatment.

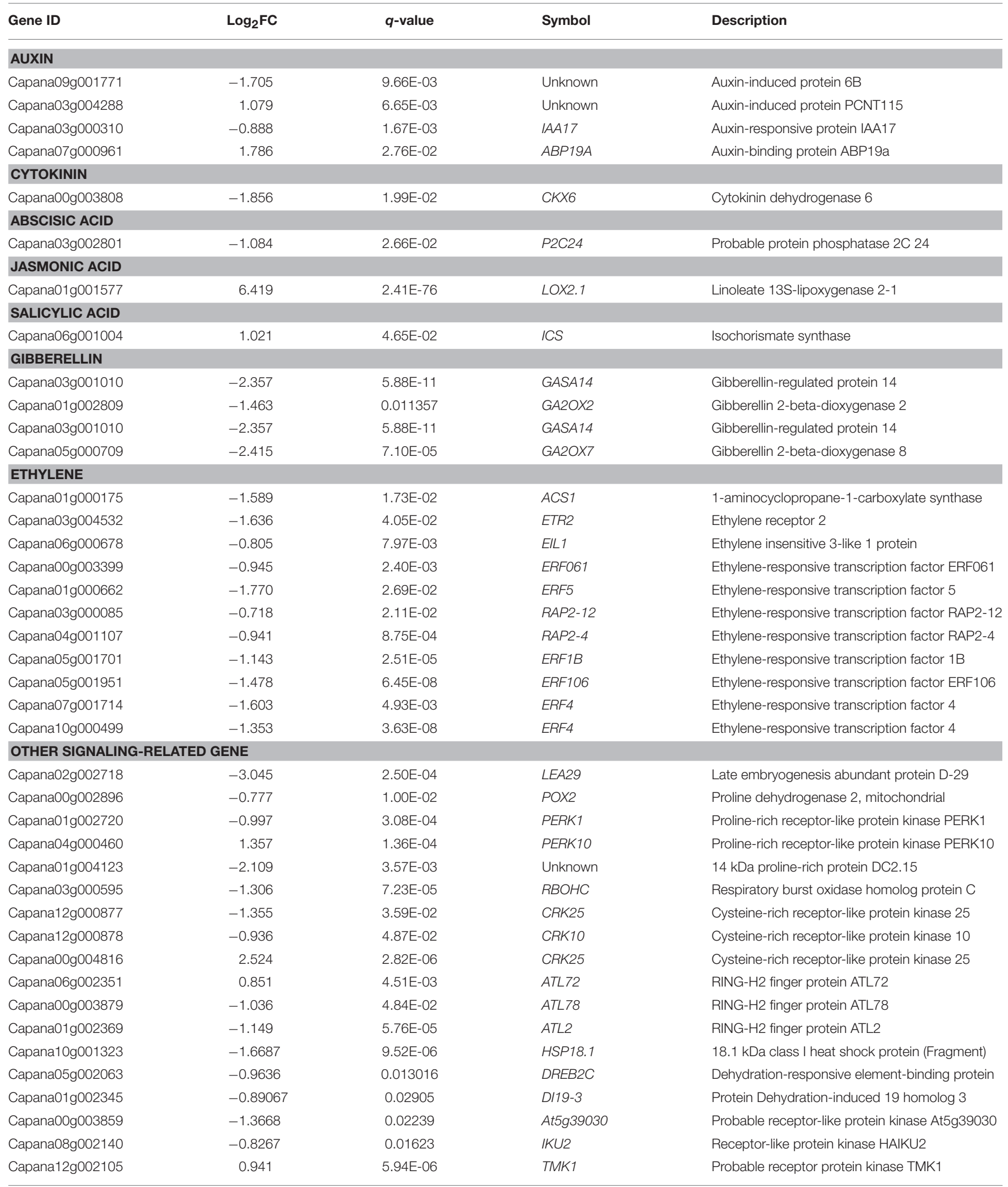

Shown are the q-value $(<0.05)$ for genes expression (comparison between EBR+Chill and Chill). 
TABLE 6 | Calcium signaling and redox-related genes expression in pepper leaves as influenced by chilling alone or in combination EBR treatment.

\begin{tabular}{|c|c|c|c|c|}
\hline Gene ID & $\log _{2} \mathrm{FC}$ & $q$-value & Symbol & Description \\
\hline \multicolumn{5}{|l|}{ CALCIUM } \\
\hline Capana00g001365 & 0.727 & $1.88 \mathrm{E}-02$ & CAS & Calcium sensing receptor \\
\hline Capana06g000284 & -1.279 & $1.77 \mathrm{E}-06$ & CIPK11 & CBL-interacting serine/threonine-protein kinase 11 \\
\hline Capana02g000787 & -1.128 & 1.06E-05 & $M C U$ & Calcium uniporter protein \\
\hline Capana03g000955 & -1.945 & 4.74E-04 & CML15 & Probable calcium-binding protein CML15 \\
\hline Capana11g000436 & -1.920 & $3.75 \mathrm{E}-07$ & CML38 & Calcium-binding protein CML38 \\
\hline Capana10g002124 & -1.084 & 8.67E-05 & CAM53 & Calmodulin-related protein \\
\hline \multicolumn{5}{|l|}{ REDOX } \\
\hline Capana09g001740 & 1.765 & 8.55E-03 & GSTX1 & Probable glutathione S-transferase \\
\hline Capana02g002747 & 2.8381 & $5.52 \mathrm{E}-06$ & PER72 & Peroxidase 72 \\
\hline Capana02g002452 & 1.149 & 4.89E-02 & CAT2 & Catalase isozyme 2 \\
\hline Capana04g000138 & 0.884 & 1.36E-03 & CDSP32 & Thioredoxin-like protein CDSP32 \\
\hline Capana01g004227 & 2.526 & $6.43 \mathrm{E}-05$ & FAO4A & Long-chain-alcohol oxidase FAO4A \\
\hline Capana00g002845 & 3.276 & 4.01E-02 & Unknown & $\mathrm{NAD}(\mathrm{P}) \mathrm{H}$ :quinone oxidoreductase \\
\hline Capana03g001927 & 1.616 & 1.49E-04 & At1g06690 & Uncharacterized oxidoreductase, chloroplastic \\
\hline
\end{tabular}

Shown are the $q$-value $(<0.05)$ for genes expression (comparison between EBR+Chill and Chill).

induction of a series of biochemical reactions to chilling stress (Table 6). In addition, EBR-treated down-regulated the genes that encoded CBL-interacting serine/threonine-protein kinase, calcium-binding protein CML, and calmodulin-related protein. It was most likely that $\mathrm{BR}$ induced calmodulin-related signaling transduction, and $\mathrm{Ca}^{2+}$ was pumped quickly out of the cells and then returned to the $\mathrm{Ca}^{2+}$ pools through intracellular $\mathrm{Ca}^{2+}$. ATPase.

Transcriptional facts (TFs) are very important for the combination of cis acting element in gene promoter elements and the mediation of the signaling pathway in response to stresses, such as WRKYs, MYBs, NACs, BHLHs, and ZFPs (Liu et al., 2008; Fujita et al., 2011; Shi and Chan, 2014; Shi et al., 2014). Several studies have shown that the expressions of C-repeat binding factors (CBFs) as master molecular switches are positively regulated by $M Y B 56, Z F P 1 / 182$, and $C A M T A 1 / 2 / 3$ and are negatively regulated by $M Y B 15$, MYBS3, WRKY34, and EIN3. The TFs specifically bind to the DRE/CRT (dehydration-responsive element/C-repeat element) cis-acting regulatory element of the promoter region of the coldresponsive genes, such as $D H N$ (dehydrin) and $R D$ (responsive to dehydration). In the present study, we found that BRs downregulated WRKY11 and WRKY40 which mediated the metabolic pathway of ABA, whereas WRKY51, which positively mediated JA- and SA-signaling was the up-regulated by EBR under chilling stress. Additionally, BRs induced up-regulation of TFs bHLH involved in AKS, which facilitated stomatal opening by triggering the phosphorylation of AKS family transcription factors. We also found that BRs positively mediated TCP19 TF, which was associated with the orchestrated regulation of ICS1 expression and the GA-mediated signaling pathway (GATA22). A study on blueberry demonstrated that the genes encoding zinc finger proteins are associated with cold acclimation (Die and Rowland, 2014). In pepper, we found that EBR up-regulated COL2 and down-regulated MGP under chilling stress. These results indicate that BRs induced different expressions of zinc finger proteins in response to chilling stress. Although it was is unknown why some TFs are up-regulated while the others are down-regulated in DEGs, we assume that TFs coordinated the network regulation of transcriptional activities in multiple pathways to increase chilling tolerance in EBR-treated pepper.

Glycosylation in plants is crucial not only for the regulation of cellular metabolism including plant hormones, secondary metabolites, and xenobiotics (Li et al., 2001), but also for the activity of several signaling molecules and defense compounds (Vogt and Jones, 2000). Sun et al. (2013) reported that glycosyltransferase could protect tobacco against salt stress, and the corresponding glycosyltransferase gene is essential for modifying cellular redox homeostasis under abiotic stress. In the present study, many glycosyltransferase genes were up-regulated by EBR (Table 7), which suggested that the up-regulation of the genes may confer cold tolerance in EBR-treated pepper. Cellulose synthase was also up-regulated, providing primary interface for plant environment interactions, and was associated the formation of cell wall for performing sophisticated strategies to respond to different environmental stresses.

Taken together, the BR-induced cold stress signals are perceived by several receptors at the cell membrane, followed by calcium and hormones molecules transduction to activate downstream stress-responsive genes in response to chilling stress. Among these, BR-induced signaling pathways might regulate transcription or directly/indirectly interact with several other signaling networks. BR promoted the change of $\mathrm{Ca}^{2+}$ in the cytoplasm- and induced a series of biochemical reactions to regulate cellular redox homeostasis related genes, such as GSTX1, PER72, and CAT2. The key transcriptional factor bHLH of the JA signaling pathway interacted with MaICE1, and then activated the expression of downstream CBF related genes in response to 
TABLE 7 | Transcription factors and post-transcription related genes expression in pepper leaves as influenced by chilling alone or in combination EBR treatment.

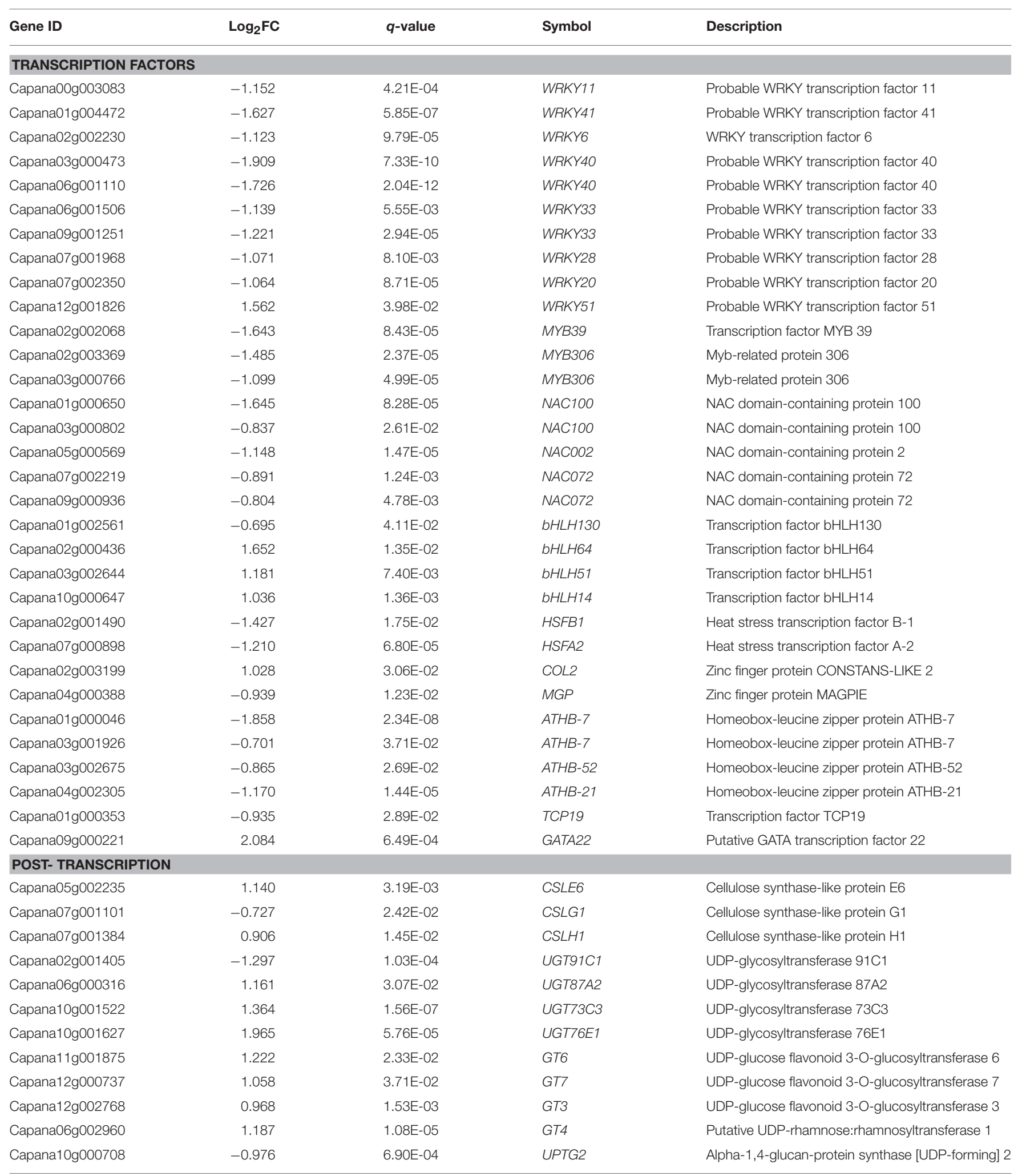

Shown are the $q$-value $(<0.05)$ for genes expression (comparison between EBR+Chill and Chill). 


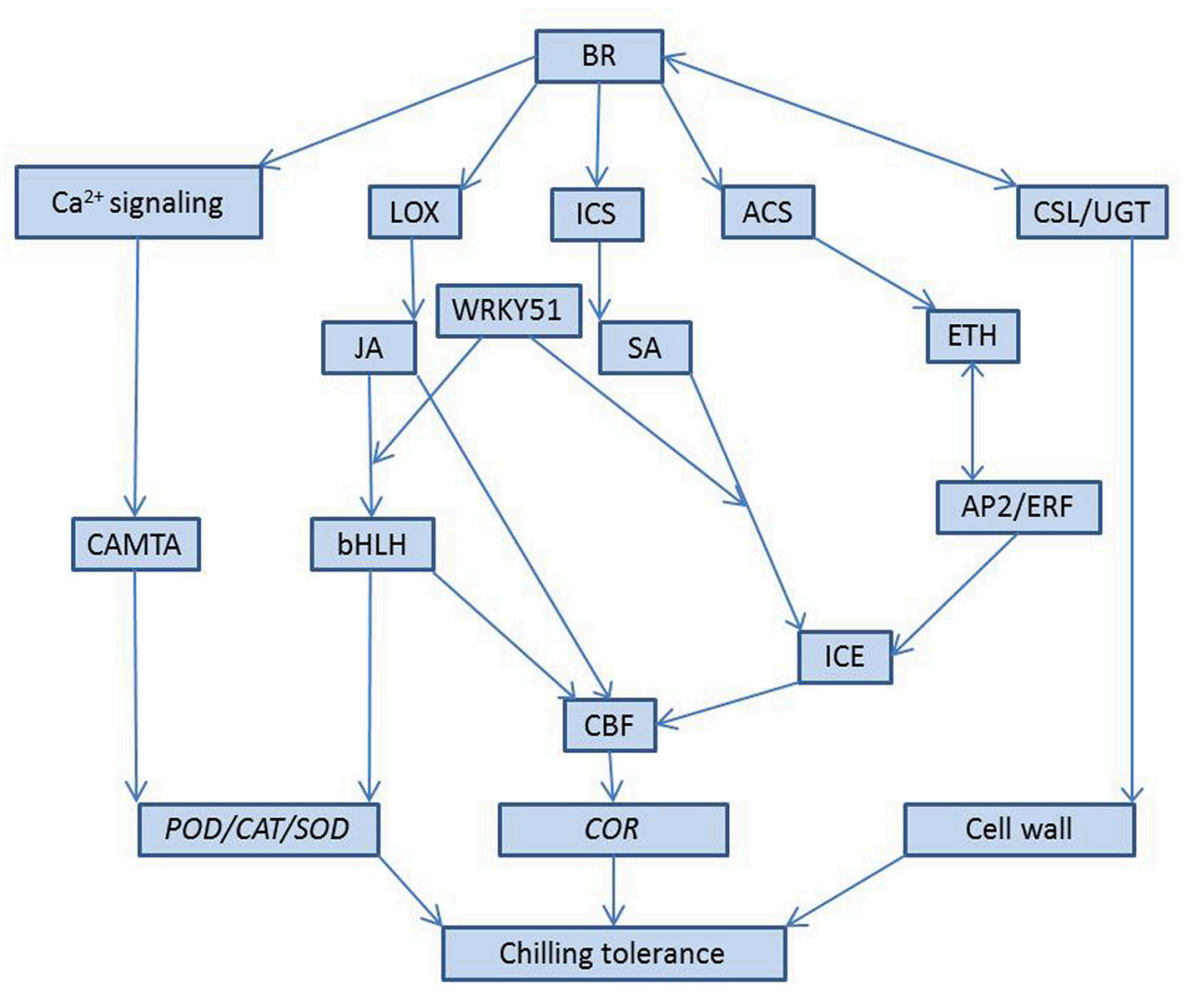

FIGURE 7 | Model depicting BR-mediated chilling tolerance via $\mathbf{C a}^{2+}$, JA, SA, ETH signaling. LOX, ICS, and ACS are jasmonic acid biosynthesis gene, salicylic acid-related gene isochorismate synthase, and ethylene biosynthesis genes ACC synthase, respectively. WRKY51, bHLH, and AP2/ERF are all transcriptional facts. CSL is cellulose synthase-like protein; UGT is UDP-glycosyltransferase. Cellular redox homeostasis related genes: GSTX1, PER72, and CAT2. CAMTA:

CaM-binding transcription activator.

chilling stress. JA, as the key upstream signal of ICE-CBF/DREB1 pathways, positively regulated cold-related gene expression in EBR-treated pepper and regulated GSTX1, PER72, and CAT2 expression. We also found that BR induced SA signaling for antistress effectsby positively regulating the expression of the CBF upstream gene ICE and that WRKY51 may be a potential point of cross-talking among JA, SA, and BR, where BR induces a subset of SA- or JA-responsive genes (Figure 7). BR negatively regulated the ETH signaling components and the TFs AP2/ERF, which indicated that BR induces the expression of cold related genes and the expression depends on the ETH signaling pathway. In addition, BR activated cellulose synthase-like protein (CSLE6 and CSLH1), which regulated the formation of a cell wall, and BR also activated UDP-glycosyltransferase genes associated with hormone metabolism, such BR glucoside, and other metabolic enzymes (e.g., PERK10, HSP, and LEA29).

\section{CONCLUSION}

In the study, we analyzed the gene expression profiles of BRs induced chilling tolerance in pepper using RNA-seq analysis. Our results showed that EBR induced 656 differently expressed genes, including 335 up-regulated and 321 down-regulated DEGs. Using GO and KEGG pathway analysis, we found that EBR application under chilling stress positively regulated photosynthesis-related genes, cellulose synthase-like protein, UDP-glycosyltransferase, and cellular redox homeostasis-related genes (GSTX1, PER72, and CAT2). Moreover, we present a model to explain the possible cross-talk of BR with SA, ETH, and JA signaling pathways under $\mathrm{BR}$-induced cold tolerance. Our study provides the first evidence of the potent roles of exogenous EBR at the transcriptional level, and the response to chilling stress in pepper involved the activation of extensive transcriptional activities, signaling transduction, and modulation of metabolic homeostasis.

\section{AUTHOR CONTRIBUTIONS}

JK and JX conceived and designed the experiments. Jie L and PY performed the experiments; Jie L, ZF, and Jia L analyzed the data. GZ and JY contributed reagents/materials/analysis tools. YG and AC helped perform the analysis with constructive discussions and language polished. JX and YG approved the final version. All authors have read and approved the final manuscript.

\section{ACKNOWLEDGMENTS}

We are grateful to the funding provided by National Natural Science Foundation of China (NSFC) (project \# 31260493). We 
also thank Mr. Dawuda M. M. and Dr. John Constable for critically reading the manuscript.

\section{SUPPLEMENTARY MATERIAL}

The Supplementary Material for this article can be found online at: http://journal.frontiersin.org/article/10.3389/fpls.2016. 01281

Table S1 | Gene primers used for qPCR validation in this study.

\section{REFERENCES}

Allen, D. J., and Ort, D. R. (2001). Impacts of chilling temperatures on photosynthesis in warm-climate plants. Trends Plant Sci. 6, 36-42. doi: 10.1016/S1360-1385(00)01808-2

Arnon, D. I. (1949). Copper enzymes in isolated chloroplasts. Polyphenoloxidase in Beta vulgaris. Plant Physiol. 24, 1-15. doi: 10.1104/pp.24.1.1

Bajguz, A. (2009). Brassinosteroid enhanced the level of abscisic acid in Chlorella vulgaris subjected to short-term heat stress. J. Plant Physiol. 166, 882-886. doi: 10.1016/j.jplph.2008.10.004

Benjamini, Y., and Hochberg, Y. (1995). Controlling the false discovery rate: a practical and powerful approach of multiple testing. J. R. Stat. Soc. Ser. B (Methodological) 57, 289-300.

Boudsocq, M., and Sheen, J. (2013). CDPKs in immune and stress signaling. Trends Plant Sci. 18, 30-40. doi: 10.1016/j.tplants.2012.08.008

Broin, M., and Rey, P. (2003). Potato plants lacking the CDSP32 plastidic thioredoxin exhibit overoxidation of the BAS1 2-cysteine peroxiredoxin and increased lipid Peroxidation in thylakoids under photooxidative stress. Plant Physiol. 132, 1335-1343. doi: 10.1104/pp.103. 021626

Chen, H., Lai, Z., Shi, J., Xiao, Y., Chen, Z., and Xu, X. (2010). Roles of arabidopsis WRKY18, WRKY40 and WRKY60 transcription factors in plant responses to abscisic acid and abiotic stress. BMC Plant Biol. 10:281. doi: 10.1186/1471-222910-281

Chen, J., Hou, K., Qin, P., Liu, H., Yi, B., Yang, W., et al. (2014). RNA-Seq for gene identification and transcript profiling of three Stevia rebaudiana genotypes. BMC Genomics 15:571. doi: 10.1186/1471-2164$15-571$

Chinnusamy, V., Zhu, J., and Zhu, J. K. (2007). Cold stress regulation of gene expression in plants. Trends Plant Sci. 12, 444-451. doi: 10.1016/j.tplants.2007.07.002

Chiwocha, S. D. S., Abrams, S. R., Ambrose, S. J., Cutler, A. J., Mary, L., Ross, A. R. S., et al. (2003). A method for profiling classes of plant hormones and their metabolites using liquid chromatography-electrospray ionization tandem mass spectrometry: an analysis of hormone regulation of thermodormancy of lettuce (Lactuca sativa L.) seeds. Plant J. 35, 405-417. doi: 10.1046/j.1365313X.2003.01800.x

Colebrook, E. H., Thomas, S. G., Phillips, A. L., and Hedden, P. (2014). The role of gibberellin signalling in plant responses to abiotic stress. J. Exp. Biol. 217(Pt 1), 67-75. doi: 10.1242/jeb.089938

Cui, J. X., Zhou, Y. H., Ding, J. G., Xia, X. J., Shi, K., Chen, S. C., et al. (2011). Role of nitric oxide in hydrogen peroxide-dependent induction of abiotic stress tolerance by brassinosteroids in cucumber. Plant Cell Environ. 34, 347-358. doi: 10.1111/j.1365-3040.2010.02248.x

Dhaubhadel, S., Browning, K. S., Gallie, D. R., and Krishna, P. (2002). Brassinosteroid functions to protect the translational machinery and heatshock protein synthesis following thermal stress. Plant J. 29, 681-691. doi: 10.1046/j.1365-313X.2002.01257.x

Dhaubhadel, S., and Krishna, P. (2008). Identification of differentially expressed genes in brassinosteroid-treated Brassica napus Seedlings. J. Plant Growth Regul. 27, 297-308. doi: 10.1007/s00344-0089056-5
Table S2 | Genes information.

Table S3 | RPKM of all genes.

Table S4 | Differential expressed genes in chilling stress with EBR-treated plant compared to only chilling treated plant.

Table S5 | Gene ontology analyses of differential expressed genes regulated by the EBR + chilling and only chilling

stress.

Table S6 | KEGG pathway enrichment of differential expressed genes regulated by the EBR + chilling and only chilling stress.

Die, J. V., and Rowland, L. J. (2014). Elucidating cold acclimation pathway in blueberry by transcriptome profiling. Environ. Exp. Bot. 106, 87-98. doi: 10.1016/j.envexpbot.2013.12.017

Divi, U. K., and Krishna, P. (2010). Overexpression of the brassinosteroid biosynthetic gene AtDWF4 in arabidopsis seeds overcomes abscisic acidinduced inhibition of germination and increases cold tolerance in transgenic seedlings. J. Plant Growth Regul. 29, 385-393. doi: 10.1007/s00344-0109150-3

Divi, U. K., Rahman, T., and Krishna, P. (2010). Brassinosteroid-mediated stress tolerance in Arabidopsis shows interactions with abscisic acid, ethylene and salicylic acid pathways. BMC Plant Biol. 10:151. doi: 10.1186/1471-222910-151

Doblin, M. S., Pettolino, F. A., Wilson, S. M., Campbell, R., Burton, R. A., Fincher, G. B., et al. (2009). A barley cellulose synthase-like CSLH gene mediates $(1,3 ; 1,4)-\beta$-d-glucan synthesis in transgenic Arabidopsis. Proc. Natl. Acad. Sci. U.S.A. 106, 5996-6001. doi: 10.1073/pnas.0902 019106

Fujita, Y., Fujita, M., Shinozaki, K., and Yamaguchi-Shinozaki, K. (2011). ABAmediated transcriptional regulation in response to osmotic stress in plants. $J$. Plant Res. 124, 509-525. doi: 10.1007/s10265-011-0412-3

Gao, Q. M., Venugopal, S., Navarre, D., and Kachroo, A. (2011). Low oleic acid-derived repression of jasmonic acid-inducible defense responses requires the WRKY50 and WRKY51 proteins. Plant Physiol. 155, 464-476. doi: 10.1104/pp.110.166876

Hao, J., Yin, Y., and Fei, S. Z. (2013). Brassinosteroid signaling network: implications on yield and stress tolerance. Plant Cell Rep. 32, 1017-1030. doi: 10.1007/s00299-013-1438-x

Henriksson, E., Olsson, A. S., Johannesson, H., Johansson, H., Hanson, J., Engström, P., et al. (2005). Homeodomain leucine zipper class I genes in Arabidopsis. Expression patterns and phylogenetic relationships. Plant Physiol. 139, 509-518. doi: 10.1104/pp.105.063461

Hu, L., Huang, Z., Liu, S., and Fu, J. (2012). Growth response and gene expression in antioxidant-related enzymes in two bermudagrass genotypes differing in salt tolerance. J. Am. Soc. Hortic. Sci. 137, 134-143.

$\mathrm{Hu}, \mathrm{Y}$., and $\mathrm{Yu}, \mathrm{D}$. (2014). BRASSINOSTEROID INSENSITIVE2 interacts with ABSCISIC ACID INSENSITIVE5 to mediate the antagonism of brassinosteroids to abscisic acid during seed germination in Arabidopsis. Plant Cell 26, 4394-4408. doi: 10.1105/tpc.114.130849

Hudson, D., Guevara, D., Yaish, M. W., Hannam, C., Long, N., Clarke, J. D., et al. (2011). GNC and CGA1 modulate chlorophyll biosynthesis and glutamate synthase (GLU1/Fd-GOGAT) expression in Arabidopsis. PLoS ONE 6:e26765. doi: 10.1371/journal.pone.0026765

Jackson, D., Culianez-Macia, F., Prescott, A. G., Roberts, K., and Martin, C. (1991). Expression patterns of myb genes from Antirrhinum flowers. Plant Cell 3, 115-125. doi: 10.1105/tpc.3.2.115

Janská, A., Marsík, P., Zelenková, S., and Ovesná, J. (2010). Cold stress and acclimation - what is important for metabolic adjustment? Plant Biol. (Stuttg). 12, 395-405. doi: 10.1111/j.1438-8677.2009.00299.x

Jansson, S. (1994). The light-harvesting chlorophyll a/b-binding proteins. Biochim. Biophys. Acta 1184, 1-19. doi: 10.1016/0005-2728(94)90148-1

Jansson, S. (1999). A guide to the Lhc genes and their relatives in Arabidopsis. Trends Plant Sci. 4, 236-240. doi: 10.1016/S1360-1385(99)01419-3 
Journot-Catalino, N., Somssich, I. E., Roby, D., and Kroj, T. (2006). The transcription factors WRKY11 and WRKY17 act as negative regulators of basal resistance in Arabidopsis thaliana. Plant Cell 18, 3289-3302. doi: 10.1105/tpc.106.044149

Kagale, S., Divi, U. K., Krochko, J. E., Keller, W. A., and Krishna, P. (2007), Brassinosteroid confers tolerance in Arabidopsis thaliana and Brassica napus to a range of abiotic stresses. Planta 225, 353-364. doi: 10.1007/s00425-00 6-0361-6

Kang, D. J., Seo, Y. J., Lee, J. D., Ishii, R., Kim, K. U., Shin, D. H., et al. (2005). Jasmonic acid differentially affects growth, ion uptake and abscisic acid concentration in salt-tolerant and salt-sensitive rice cultivars. J. Agron. Crop Sci. 191, 273-282. doi: 10.1111/j.1439-037X.2005.00153.x

Kim, S. K., Park, H. Y., Jang, Y. H., Lee, J. H., and Kim, J. K. (2013). The sequence variation responsible for the functional difference between the CONSTANS protein, and the CONSTANS-like (COL) 1 and COL2 proteins, resides mostly in the region encoded by their first exons. Plant Sci. 199-200, 71-78. doi: 10.1016/j.plantsci.2012.09.019

Li, J., Yang, P., Gan, Y., Yu, J., and Xie, J. (2015a). Brassinosteroid alleviates chilling-induced oxidative stress in pepper by enhancing antioxidation systems and maintenance of photosystem II. Acta Physiol. Plant. 37, 1-11. doi: 10.1007/s11738-015-1966-9

Li, J., Yang, P., Xie, J., and Yu, J. (2015b). Effects of 24-epibrassinolide on growth and antioxidant enzymes system in pepper roots under chilling stress. J. Nucl. Agric. Sci. 29, 1001-1008. doi: 10.11869/j.issn.100-8551.2015.05.1001

Li, Y., Baldauf, S., Lim, E. K., and Bowles, D. J. (2001). Phylogenetic analysis of the UDP-glycosyltransferase multigene family of Arabidopsis thaliana. J. Biol. Chem. 276, 4338-4343. doi: 10.1074/jbc.M007447200

Liscum, E., and Reed, J. W. (2002). Genetics of Aux/IAA and ARF action in plant growth and development. Plant Mol. Biol. 49, 387-400. doi: 10.1023/A:1015255030047

Liu, H., Zhang, H., Yang, Y., Li, G., Yang, Y., Wang, X., et al. (2008). Functional analysis reveals pleiotropic effects of rice RING-H2 finger protein gene OsBIRF1 on regulation of growth and defense responses against abiotic and biotic stresses. Plant Mol. Biol. 68, 17-30. doi: 10.1007/s11103-0089349-x

Liu, J., Gao, H., Wang, X., Zheng, Q., Wang, C., Wang, X., et al. (2014). Effects of 24-epibrassinolide on plant growth, osmotic regulation and ion homeostasis of salt-stressed canola. Plant Biol. (Stuttg). 16, 440-450. doi: 10.1111/plb. 12052

Liu, T., Zhu, S., Tang, Q., and Tang, S. (2015). Genome-wide transcriptomic profiling of ramie (Boehmeria nivea L. Gaud) in response to cadmium stress. Gene 558, 131-137. doi: 10.1016/j.gene.2014.12.057

Livak, K. J., and Schmittgen, T. D. (2001). Analysis of relative gene expression data using real-time quantitative PCR and the 2(-Delta Delta C(T)) Method. Methods 25, 402-408. doi: 10.1006/meth.2001.1262

Lu, X., Kim, H., Zhong, S., Chen, H., Hu, Z., and Zhou, B. (2014). De novo transcriptome assembly for rudimentary leaves in Litchi chinesis Sonn. and identification of differentially expressed genes in response to reactive oxygen species. BMC Genomics 15:805. doi: 10.1186/1471-2164-15-805

Marino, D., Dunand, C., Puppo, A., and Pauly, N. (2012). A burst of plant NADPH oxidases. Trends Plant Sci. 17, 9-15. doi: 10.1016/j.tplants.2011.10.001

Mazorra, L. M., Nunez, M., Hechavarria, M., Coll, F., and Sanchez-Blanco, M. J. (2002). Influence of brassinosteroids on antioxidant enzymes activity in tomato under different temperatures. Biol. Plant. 45, 593-596. doi: 10.1023/A:1022390917656

Mortazavi, A., Williams, B. A., McCue, K., Schaeffer, L., and Wold, B. (2008). Mapping and quantifying mammalian transcriptomes by RNA-Seq. Nat. Methods 5, 621-628. doi: 10.1038/nmeth.1226

Pantin, F., Monnet, F., Jannaud, D., Costa, J. M., Renaud, J., Muller, B., et al. (2013). The dual effect of abscisic acid on stomata. New Phytol. 197, 65-72. doi: $10.1111 /$ nph. 12013

Peng, Q., and Zhou, Q. (2009). The endogenous hormones in soybean seedlings under the joint actions of rare earth element $\mathrm{La}(\mathrm{III})$ and ultraviolet-B stress. Biol. Trace Elem. Res. 132, 270-277. doi: 10.1007/s12011-009-8404-z

Prasad, T. K., and Cline, M. G. (1987). The role of gravity in apical dominance: effects of clinostating on shoot inversion-induced ethylene production, shoot elongation, and lateral bud growth. Plant Physiol. 83, 505-509. doi: 10.1104/pp.83.3.505
Qin, C., Yu, C., Shen, Y., Fang, X., Chen, L., Min, J., et al. (2014). Whole-genome sequencing of cultivated and wild peppers provides insights into Capsicum domestication and specialization. Proc. Natl. Acad. Sci. U.S.A. 111, 5135-5140. doi: 10.1073/pnas.1400975111

Ren, L., Sun, J., Chen, S., Gao, J., Dong, B., Liu, Y., et al. (2014). A transcriptomic analysis of Chrysanthemum nankingense provides insights into the basis of low temperature tolerance. BMC Genomics 15:844. doi: 10.1186/1471-216415-844

Richter, R., Behringer, C., Muller, I. K., and Schwechheimer, C. (2010). The GATA-type transcription factors GNC and GNL/CGA1 repress gibberellin signaling downstream from DELLA proteins and PHYTOCHROMEINTERACTING FACTORS. Genes Dev. 24, 2093-2104. doi: 10.1101/gad. 594910

Sanghera, G. S., Wani, S. H., Hussain, W., and Singh, N. B. (2011). Engineering cold stress tolerance in crop plants. Curr. Genomics 12, 30-43. doi: $10.2174 / 138920211794520178$

Shi, H., and Chan, Z. (2014). The cysteine2/histidine2-type transcription factor ZINC FINGER OF ARABIDOPSIS THALIANA 6-activated CREPEAT-BINDING FACTOR pathway is essential for melatonin-mediated freezing stress resistance in Arabidopsis. J. Pineal Res. 57, 185-191. doi: 10.1111/jpi.12155

Shi, H., Ye, T., and Chan, Z. (2014). Comparative proteomic responses of two bermudagrass (Cynodon dactylon (L). Pers.) varieties contrasting in drought stress resistance. Plant Physiol. Biochem. 82, 218-228. doi: 10.1016/j.plaphy.2014.06.006

Sun, Y. G., Wang, B., Jin, S. H., Qu, X. X., Li, Y. J., and Hou, B. K. (2013). Ectopic expression of Arabidopsis glycosyltransferase UGT85A5 enhances salt stress tolerance in tobacco. PLOS ONE 8:e59924. doi: 10.1371/journal.pone.0059924

Takahashi, Y., Ebisu, Y., Kinoshita, T., Doi, M., Okuma, E., Murata, Y., et al. (2013). bHLH transcription factors that facilitate $\mathrm{K}(+)$ uptake during stomatal opening are repressed by abscisic acid through phosphorylation. Sci. Signal. 6, ra48. doi: $10.1126 /$ scisignal.2003760

Vogt, T., and Jones, P. (2000). Glycosyltransferases in plant natural product synthesis: characterization of a supergene family. Trends Plant Sci. 5, 380-386. doi: 10.1016/S1360-1385(00)01720-9

Wang, J., Li, B., Meng, Y., Ma, X., Lai, Y., Si, E., et al. (2015). Transcriptomic profiling of the salt-stress response in the halophyte Halogeton glomeratus. BMC Genomics 16:169. doi: 10.1186/s12864-015-1373-Z

Wang, L., Feng, Z., Wang, X., Wang, X., and Zhang, X. (2010). DEGseq: an $\mathrm{R}$ package for identifying differentially expressed genes from RNA-seq data. Bioinformatics 26, 136-138. doi: 10.1093/bioinformatics/btp612

Wang, X., Gao, J., Zhu, Z., Dong, X., Wang, X., Ren, G., et al. (2015). TCP transcription factors are critical for the coordinated regulation of isochorismate synthase 1 expression in Arabidopsis thaliana. Plant J. 82, 151-162. doi: $10.1111 /$ tpj. 12803

Welch, D., Hassan, H., Blilou, I., Immink, R., Heidstra, R., and Scheres, B. (2007). Arabidopsis JACKDAW and MAGPIE zinc finger proteins delimit asymmetric cell division and stabilize tissue boundaries by restricting SHORT-ROOT action. Genes Dev. 21, 2196-2204. doi: 10.1101/gad.440307

Werner, T., Motyka, V., Laucou, V., Smets, R., Van Onckelen, H., and Schmülling, T. (2003). Cytokinin-deficient transgenic arabidopsis plants show multiple developmental alterations indicating opposite functions of cytokinins in the regulation of shoot and root meristem activity. Plant Cell 15, 2532-2550. doi: $10.1105 /$ tpc. 014928

Xia, X. J., Wang, Y. J., Zhou, Y. H., Tao, Y., Mao, W. H., Shi, K., et al. (2009). Reactive oxygen species are involved in brassinosteroid-induced stress tolerance in cucumber. Plant Physiol. 150, 801-814. doi: 10.1104/pp.109.138230

Xie, C., Mao, X., Huang, J., Ding, Y., Wu, J., Dong, S., et al. (2011). KOBAS 2.0: a web server for annotation and identification of enriched pathways and diseases. Nucleic Acids Res. 39, W316-W322. doi: 10.1093/nar/ gkr483

Young, M. D., Wakefield, M. J., Smyth, G. K., and Oshlack, A. (2010). Gene ontology analysis for RNA-seq: accounting for selection bias. Genome Biol. 11:R14. doi: 10.1186/gb-2010-11-2-r14

Yu, X., Li, L., Zola, J., Aluru, M., Ye, H., Foudree, A., et al. (2011). A brassinosteroid transcriptional network revealed by genome-wide identification of BESI target genes in Arabidopsis thaliana. Plant J. 65, 634-646. doi: 10.1111/j.1365313X.2010.04449.x 
Yuan, G. F., Jia, C. G., Li, Z., Sun, B., Zhang, L. P., Liu, N., et al. (2010). Effect of brassinosteroids on drought resistance and abscisic acid concentration in tomato under water stress. Sci. Hortic. 126, 103-108. doi: 10.1016/j.scienta.2010.06.014

Yuan, X., Deng, K. Q., Zhao, X. Y., Wu, X. J., Qin, Y. Z., Tang, D. Y., et al. (2007). A calcium-dependent protein kinase is involved in plant hormone signal transduction in Arabidopsis. J. Plant Physiol. Mol. Biol. 33, 227-234.

Zhang, L., Li, Y., Xing, D., and Gao, C. (2009). Characterization of mitochondrial dynamics and subcellular localization of ROS reveal that HsfA2 alleviates oxidative damage caused by heat stress in Arabidopsis. J. Exp. Bot. 60, 2073-2091. doi: 10.1093/jxb/erp078
Conflict of Interest Statement: The authors declare that the research was conducted in the absence of any commercial or financial relationships that could be construed as a potential conflict of interest.

Copyright (c) 2016 Li, Yang, Kang, Gan, Yu, Calderón-Urrea, Lyu, Zhang, Feng and Xie. This is an open-access article distributed under the terms of the Creative Commons Attribution License (CC BY). The use, distribution or reproduction in other forums is permitted, provided the original author(s) or licensor are credited and that the original publication in this journal is cited, in accordance with accepted academic practice. No use, distribution or reproduction is permitted which does not comply with these terms. 